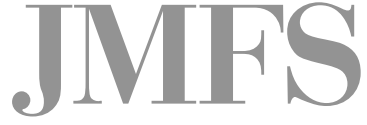

Journal of Management and Financial Sciences
Volume XI

Issue 33 (September 2018)

pp. 117-145

Warsaw School of Economics

Collegium of Management and Finance

Paweł Sobczak

WSB University, Poland

\title{
Structural Analysis of Network Connections of Koleje Małopolskie sp. z o.o. as a Significant Element of the Management of the Transport Company
}

\begin{abstract}
Public transport is one of the most important elements of the economy and social space development in which it is located. Issues related to its proper organisation are very often the responsibility of transport companies, which must operate and carry out their activities within the principles of the economy, that is, the principles of managing a service company. An important element of an efficient and effective functioning of a transport company is the quality of the provided services. An element significantly affecting the quality of the services offered is, inter alia, an appropriately organised network of connections offered by the carrier. This network in an efficient and interference-resistant manner must facilitate transport in a given area. In the article, using the graph theory and the simulation method, the structure of network connections of a railway carrier operating in the Małopolska region (Koleje Małopolskie sp. $z$ o.o.) in Poland was analysed. The aim of the analysis was to obtain information about the current condition and parameters of the network offered by the carrier. The conducted analysis made it possible to assess the current state as well as within the conducted research and it proposed to modify the existing network of connections in order to improve its parameters. The carrier's network is used, inter alia, to co-create public transport in the region, and the structure of the connections network and its appropriate planning can have a significant impact on the functioning of the company.
\end{abstract}

Keywords: public transport, rail transport, transport networks, Koleje Małopolskie sp. z o.o., railway network

JEL Classification Codes: L92 


\section{Introduction}

Sustainable transport is now one of the European Union's priorities in the area of its current activities. The current transport situation in Poland and Europe connected with a very large use of individual transport for the implementation of everyday displacements and related negative effects in the form of, among others, environmental pollution, noise, smog and the effects of road accidents (e.g. accidents and related injuries, death, etc.) cause the European Union, as part of its transport policy, to try to limit the share of car transport (including the individual one) to other modes of transport.

The above activities are particularly important in large cities or agglomerations such as Kraków. In Kraków, actions have been taken for many years to overcome the negative effects of transport (mainly individual) on the environment and cultural heritage (including monuments), which the city is famous for. These activities include: designing bus corridors, charges for parking the car in the city centre (which also aims to discourage the use of individual transport), separation of corridors for trams, etc. Obivously, these activities have the effect of reducing the share of individual transport in public transport, but these are not sufficient actions. In connection with the above, the city authorities and voivodships undertake further actions in the field of promoting public transport for individual transport.

In addition, for several years in Poland, based on global trends, measures have been taken to diversify the modes of transport that implement public transport (both in the agglomeration and the region), by including in the public transport organization the so-called urban or regional rail transport. This is to increase transport accessibility in Poland.

Activities of this type were also undertaken in the Małopolska region by appointing the railway carrier - Koleje Małopolskie sp. z o.o. Based on the existing railway infrastructure, this carrier has created a network of connections in which it provides transport services. The layout of this network and its structure are a very important element affecting the level and quality of services provided, therefore, it is important and recommended to analyse its structure using currently used methods of network analysis - this analysis is the main goal of the article. The analysis was carried out using the so-called graph theory. The article, which is an introductory article on the analysis of the transport network of the Koleje Małopolskie sp z o.o. carrier, focused on the analysis of the connection network developed by the carrier and also after that analysis it proposed modifications the implementation of which may contribute to the improvement of network parameters and thus, the performance of the company, such as increasing the number of travelers. 


\section{Koleje Małopolskie}

Koleje Małopolskie is a company that was established by the Authorities of the Małopolskie Voivodship in December 2013 to provide public services in the field of public transport by ensuring the effective organization and operation of passenger rail traffic in the Małopolskie Voivodship [Koleje Małopolskie, on-line, 28/03/2018]. In order to carry out its activities in June 2014, the company obtained a license to perform rail transport of persons No. WPO / 236/2014 [Koleje Małopolskie, on-line, 28/03/2018], in August 2014 obtained a European security certificate and in October 2014, a national safety certificate [Koleje Małopolskie, on-line, 28/03/2018].

As part of its activities, Koleje Małopolskie is tasked with providing transport services in the Małopolskie voivodship, but what is important, these services must also be provided in accordance with the principles of the free market economy. This situation causes the company, while undertaking its activities, to be forced to function as any enterprise whose primary goal is to develop and improve the quality of services provided, but also to obtain an economic benefit from the services provided. Thanks to these activities, the company will be able to provide services at a competitive level in relation to other carriers (operating, for example, in other branches, such as cars) and increase its market share.

Currently, the company has had only a few years of activity, but in this period has made its intensive development and at the moment already has a significant number of transported passengers and began to increase its transport performance. The detailed data on the number of transported passengers and transport performance is presented in Table 1 and graphically in Figures 1 and 2.

Table 1. The number of transported passengers and transport performance made by Koleje Małopolskie in years 2014-2017

\begin{tabular}{|l|c|c|c|c|}
\cline { 2 - 5 } \multicolumn{1}{c|}{} & \multicolumn{4}{c|}{ Year } \\
\cline { 2 - 5 } \multicolumn{1}{c|}{} & 2014 & 2015 & 2016 & 2017 \\
\hline Number of transported passengers [m] & 0.026136 & 1.801765 & 4.788024 & 5.730428 \\
\hline Transport performance [m pass. km] & 0.517178 & 28.399650 & 87.086964 & 168.350904 \\
\hline
\end{tabular}

Source: Statistical data received electronically from the Office of Rail Transport on January 16, 2018.

As shown in Table 1 and in Figures 1 and 2, Koleje Małopolskie since the beginning of its activity has shown an almost linear increase in the number of passengers and transport performance. This proves that the carrier is very well received by the passengers, which is certainly influenced by a lot of factors (including the effect of a new "player" on the market, greater flexibility of travel by increasing the choice of transport modes, the modern fleet used by the carrier, etc.). 
Figure 1. The number of passengers transported by Koleje Małopolskie in the years 2014-2017

Number of transported passengers

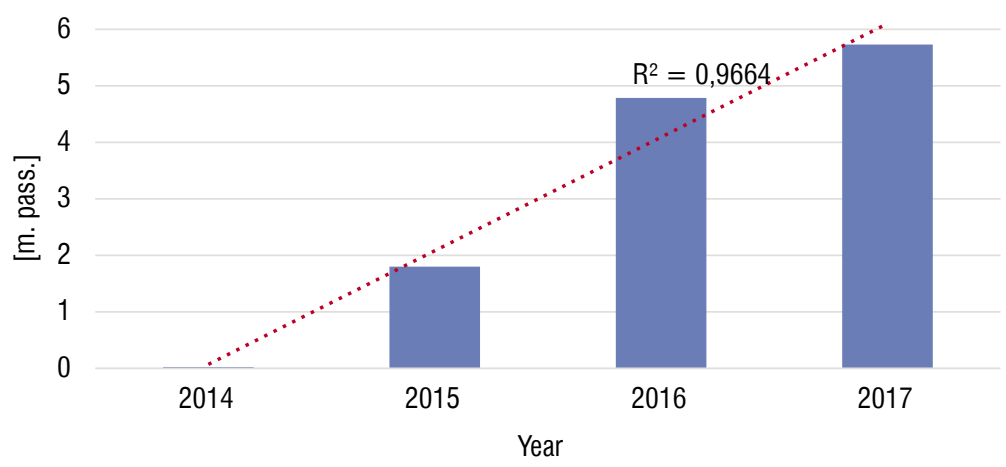

Source: the author's own elaboration based on Table 1.

Figure 2. The transport performance made by Koleje Małopolskie in the years 2014-2017

Transport performance

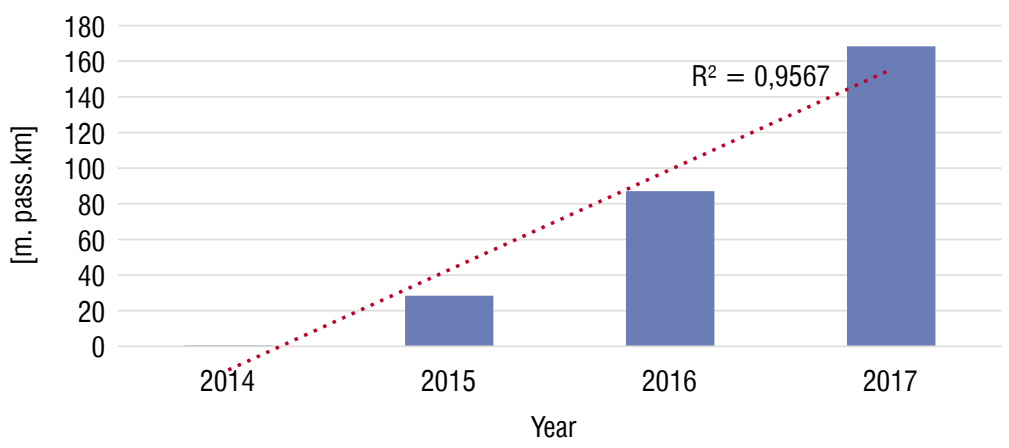

Source: the author's own elaboration based on Table 1.

However, in order to maintain a very good trend of increasing the number of passengers carried and the share in the local transport services market, further measures are necessary. One of such activities may be activities related to matching the offered network of connections with the needs and requirements of travelers as well as technical capabilities of the existing railway infrastructure managed by PKP PLK S.A., in whose infrastructure services are provided by the analysed carrier. On the absis of the above-mentioned railway network and in the voivodship, other rail carriers also provide their services, and what is more important, their services are also provided by carriers from other modes of transport. In connection with the above, the network of connections offered by the carrier should enable it to provide services both at a high level of reliability as well as at a high organisational level. For this purpose, the carrier's network should be analysed for some possible modifications to improve it. Undoubtedly, the structure of the connection network is not the only element that affects the quality and level of services provided. Many factors influence it, including nature of the area around 
the given node (whether the existing facilities there may be generators of increased passenger traffic, e.g. hospitals, schools, universities, etc.), what is the capacity between the given node and neighboring ones, what is the travel speed as well as what is the technical level and layout of competitive transport branches in the local area. These are very important factors affecting the traffic flows generated and the choice of means of transport by the traveler, however, taking into account all these factors is a difficult and complicated issue, and sometimes even impossible to tackle due to, for example, a lack of access to the required reliable technical and statistical data (e.g. due to the so-called "business secret"). Taking into account the above-mentioned factors, the article focuses on the analysis of the carrier's transport network of connections, which is presented in Figure 3.

Figure 3 The carrier's current network of connections

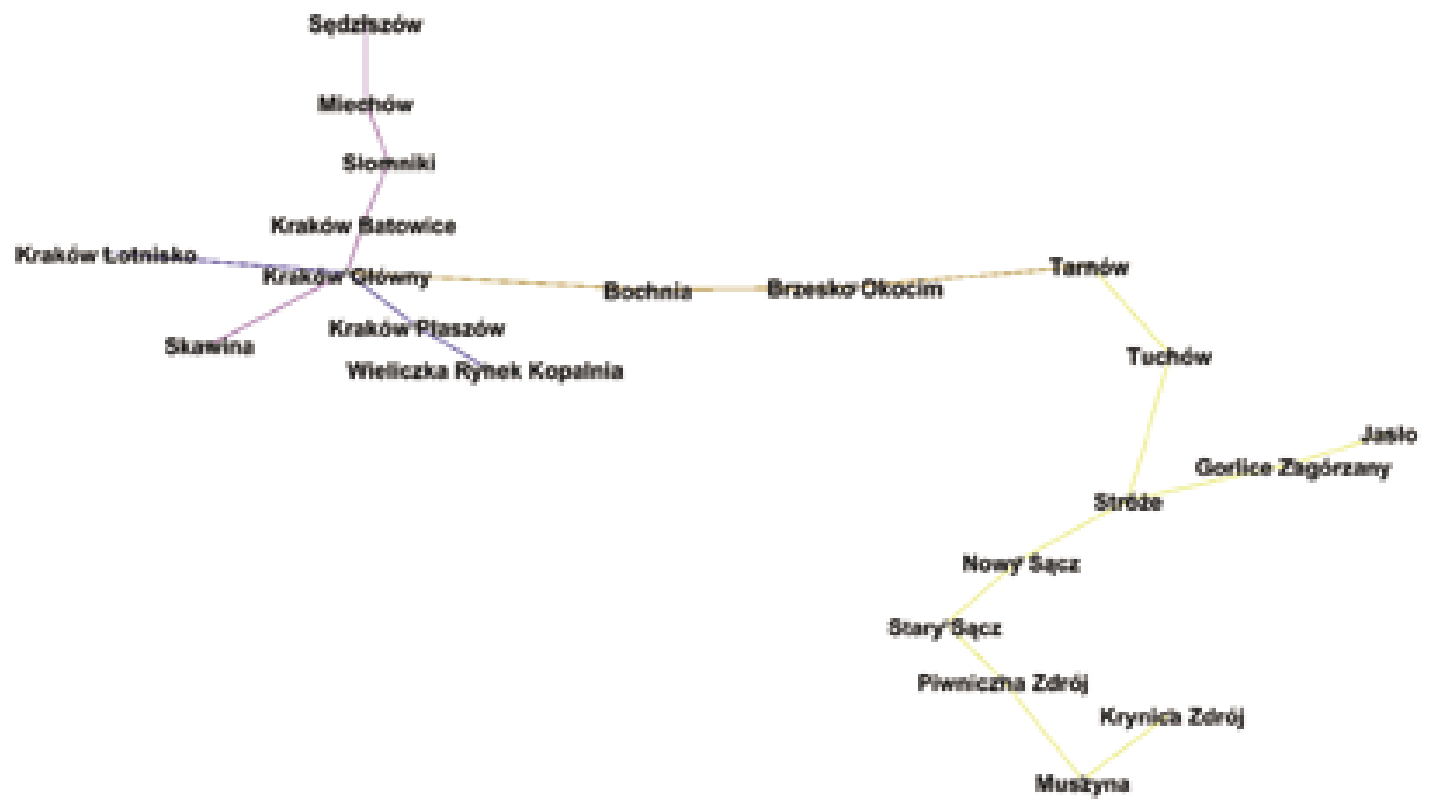

Source: the author's own elaboration based on the railway infrastructure map in Poland and information placed on the website of the Małopolska Railways on April 5, 2018.

As shown in Figure 3, the connection network of Koleje Małopolskie was divided into 4 lines (depicted by 4 connection colours: blue, violet, orange and yellow).

The analysis of the above network will provide information about its individual parameters and enable to obtain information which nodes (stations) play a key role in the network and what kind of possible changes or modifications can be made to the network in order to improve it for better performance, which in the future may be translated into a further increase in the number of passengers travelling by this carrier.

The carrier's network of connections to obtain the above information may be analysed, among others using the graph theory. 


\section{Graph theory in the analysis of transport networks}

The graph theory is used to analyse various types of networks, mainly social networks [Amaral L. et al., 2000, Newman M. et al., 2002, Arenas A. et al., 2003], but it is also used to analyse neural networks [Sporns O., 2002; Stam C. et al., 2007; Bullmore E. et al., 2009], and biological ones [Rual JF. Et al., 2005]. The analysis possibilities using the graph theory of the previously mentioned networks are also presented in [Newman M., 2010]. The graph theory is also used to analyse computer networks [Valverde S. et al., 2003] and transport networks [Newman M., 2010; Wilkinson S. et al., 2012; Li H. et al., 2014; Ouyang M. and others, 2015; Tarapata Z., 2015; Dunn, Wilkinson 2017].

The information on the role of individual nodes in the network obtained from the analysis allow initially estimating which of the points are particularly sensitive to potential threats or attacks [Newman M., 2010, Tarapata Z., 2015]. Most of the measures and calculations used also allow obtaining information which of the nodes of the network plays the main role or are a specific centre of the analysed network. According to the information contained in the literature [Tarapata Z., 2015], each network can be described as a set of nodes and links between them:

$$
G=\langle V, E\rangle
$$

where:

$\mathrm{V}$ - set of nodes;

$\mathrm{E}$ - set of connections between nodes.

Obviously, for every analysed network there is a dependence that:

$$
|V|=N,|E|=M
$$

where:

$N$ - number of nodes in the network;

$M$ - number of edges (connections) in the network.

The most frequently used indicators include those described in the literature [for example: Newman M., 2010, Tarapata Z., 2015], are:

1) Normalized degree dci i-th network node:

$$
d c_{i}=\frac{k_{i}}{N-1}
$$

where:

$k_{i}$ - the degree of the $i$-th node in the network (the number of network node connections with other nodes);

$N$ - number of nodes in the network. 
The greater the value of the $\mathrm{dc}_{\mathrm{i}}$ index for the $\mathrm{i}$-th node, the node is more important in the network or closer to its centre.

2) Eccentricity $e c_{i} i$-th network node.

$$
e c_{i}=\max _{j \in V} d_{i j}
$$

where:

$d_{i j}$ - the number of links between nodes that occurs on the shortest path between node $i$ and $j$.

The lower the value of the $e c_{i}$ index for the $i$-th node, the node is more important in the network or closer to its centre.

3) Radius rci i-th network node:

$$
r c_{i}=\frac{1}{\max _{j \in V} d_{i j}}=\frac{1}{e c_{i}}
$$

where:

$d_{i j}$ - the number of links between nodes that occurs on the shortest path between node $i$ and $j$;

$e c_{i}$ - Eccentricity for the $i$-th network node.

The greater the value of the $r c_{i}$ index for the $i$-th node, the node is more important in the network or closer to its centre.

4) Closeness $c c_{i}$ :

$$
c c_{i}=\frac{N-1}{\sum_{j \in V} d_{i j}}
$$

where:

$N$ - number of nodes in the network;

$d_{i j}$ - the number of links between nodes that occurs on the shortest path between node $i$ and $j$.

5) Beetweeness $b c_{i} i$-th network node:

$$
b c_{i}=\sum_{l \in V} \sum_{k \neq l \in V} \frac{p_{l, i, k}}{p_{l, k}}
$$

where:

$p_{l, i, k^{-}}$number of connections with the shortest number of constraints between nodes $l$ and $k$ (containing node $\mathrm{i}$ );

$p_{l, k}$ - number of connections with the shortest number of constraints between nodes $l$ and $k$ (not including node $\mathrm{i}$ ).

The greater the value of the $b c_{i}$ index for the $i$-th node, the node is more important in the network or closer to its centre. 
6) Clusterization $g c_{i} i$-th network node:

$$
g c_{i}=\frac{2 E_{i}}{k_{i}\left(k_{i}-1\right)}, k_{i}>1
$$

where:

$E_{i}$ - the number of bonds between the nodes that are closest to (neighbors) of the $i$-th node;

$k_{i}$ - degree of the $i$-th node in the network (number of network node connections with other nodes).

The greater the value of the $g c_{i}$ index for the $i$-th node, the node is more important in the network or closer to its centre.

Formulas (2)-(8) describe the parameters of individual network nodes, but in addition to them, coefficients are often used to determine the parameters of the entire analysed network. These are [Newman M., 2010, Tarapata Z., 2015]:

7) Average shortest paths length $L$ :

$$
L=\frac{1}{N(N-1)} \sum_{i \neq j \in V} d_{i j}
$$

where:

$N$ - number of nodes in the network;

$d_{i j}$ - the number of links between nodes that occurs on the shortest path between node $i$ and $j$.

The shorter the average shortest paths length is, the better the analysed network is.

8) Clusterization coefficent $C$ :

$$
C=\frac{1}{N} \sum_{i \in V} g c_{i}
$$

where:

$N$ - number of nodes in the network;

$g c_{i}$ - clusterization.

The higher the Clusterization coefficent value, the better the analysed network is.

9) Diameter $D$ :

$$
D=\max _{i \in V} e c_{i}
$$

where:

$e c_{i}$ - Eccentricity for the $i$-th network node.

The smaller the network diameter, the better the network.

10) Radius of a network $R$ :

$$
R=\min _{i \in V} e c_{i}
$$


where:

$e c_{i}$ - Eccentricity for the $i$-th network node.

The smaller the Radius of a network, the better the network.

11) Average nodes degree $\bar{k}$ :

where:

$$
\bar{k}=\frac{1}{N} \sum_{i \in V} k_{i}
$$

$N$ - number of nodes in the network;

$k_{i}$ - degree of the $i$-th node in the network (number of network node connections with other nodes).

The calculation of the values of the above indicators makes it possible to assess the dependencies between individual network nodes as well as to determine what type of network we are dealing with and which network or which nodes in the network play an important role. In networks, there are practically no situations in which all network nodes have the same degree of "importance".

Each network has key nodes that are more than others responsible for the proper functioning of the entire network or are important in it for providing services in a key area. Determining these nodes and their locations allows drawing conclusions about the current state of the network and its resistance to possible interference. This information also allows entering or proposing improvements to the analysed network.

\section{Analysis of the Koleje Małopolskie network}

The network of connections of Koleje Małopolskie was analysed using the graph theory. The Freeware Gephi software was used to perform the calculation and visualization of the network.

For the connection network shown in Figure 3, the parameters of individual nodes (stations) were calculated and presented in Table 2.

Table 2. Parameters of individual network nodes (stations) for the analysed network of connections of Koleje Małopolskie

\begin{tabular}{|l|l|c|c|c|c|c|c|}
\hline \multicolumn{1}{|c|}{ City (Node) } & $\begin{array}{c}\text { Normalized } \\
\text { degree }\end{array}$ & Eccentricity & \multicolumn{1}{c|}{ Radius } & $\begin{array}{c}\text { Closeness } \\
\text { centrality }\end{array}$ & $\begin{array}{c}\text { Betweeness } \\
\text { centrality }\end{array}$ & Clustering & Eigencentrality \\
\hline Kraków Lotnisko & 0.00952381 & 65 & 0.015384615 & 0.033903778 & 0 & 0 & 0.007164535 \\
\hline Kraków Zakliki & 0.019047619 & 63 & 0.015873016 & 0.036319613 & 0.037728938 & 0 & 0.017865051 \\
\hline Kraków Olszanica & 0.019047619 & 64 & 0.015625 & 0.035081858 & 0.019047619 & 0 & 0.012933559 \\
\hline Kraków Młynówka & 0.019047619 & 62 & 0.016129032 & 0.037620924 & 0.056043956 & 0 & 0.035020425 \\
\hline Kraków Łobzów & 0.019047619 & 61 & 0.016393443 & 0.038989974 & 0.073992674 & 0 & 0.137585316 \\
\hline Kraków Główny & 0.057142857 & 60 & 0.016666667 & 0.040431267 & 0.35 & 0 & 0.736025324 \\
\hline Kraków Płaszów & 0.057142857 & 58 & 0.017241379 & 0.042151746 & 0.412087912 & 0 & 0.899403844 \\
\hline
\end{tabular}




\begin{tabular}{|c|c|c|c|c|c|c|c|}
\hline City (Node) & $\begin{array}{c}\text { Normalized } \\
\text { degree }\end{array}$ & Eccentricity & Radius & $\begin{array}{l}\text { Closeness } \\
\text { centrality }\end{array}$ & $\begin{array}{c}\text { Betweeness } \\
\text { centrality }\end{array}$ & Clustering & Eigencentrality \\
\hline Kraków Prokocim & 0.057142857 & 57 & 0.01754386 & 0.042909685 & 0.42014652 & 0 & 0.742916948 \\
\hline Kraków Bieżanów & 0.057142857 & 56 & 0.017857143 & 0.043659044 & 0.478388278 & 0 & 0.518118741 \\
\hline $\begin{array}{l}\text { Kraków Bieżanów } \\
\text { Drożdżownia }\end{array}$ & 0.019047619 & 57 & 0.01754386 & 0.04194966 & 0.056043956 & 0 & 0.104727468 \\
\hline Wieliczka Bogucice & 0.019047619 & 58 & 0.017241379 & 0.040338071 & 0.037728938 & 0 & 0.029752948 \\
\hline Wieliczka Park & 0.019047619 & 59 & 0.016949153 & 0.038817006 & 0.019047619 & 0 & 0.014729164 \\
\hline Wieliczka Rynek Kopalnia & 0.00952381 & 60 & 0.016666667 & 0.03737985 & 0 & 0 & 0.007395471 \\
\hline Kraków Zabłocie & 0.076190476 & 59 & 0.016949153 & 0.041387466 & 0.428937729 & 0 & 1 \\
\hline Skawina & 0.00952381 & 65 & 0.015384615 & 0.033535612 & 0 & 0 & 0.007134922 \\
\hline Kraków Sidzina & 0.019047619 & 64 & 0.015625 & 0.03468781 & 0.019047619 & 0 & 0.012656507 \\
\hline Kraków Swoszowice & 0.019047619 & 63 & 0.015873016 & 0.035897436 & 0.037728938 & 0 & 0.0156532 \\
\hline Kraków Sanktuarium & 0.019047619 & 62 & 0.016129032 & 0.037168142 & 0.056043956 & 0 & 0.019669449 \\
\hline Kraków Łagiewniki & 0.019047619 & 61 & 0.016393443 & 0.03850385 & 0.073992674 & 0 & 0.042262614 \\
\hline Kraków Podgórze & 0.019047619 & 60 & 0.016666667 & 0.03990878 & 0.091575092 & 0 & 0.182326965 \\
\hline Kraków Batowice & 0.019047619 & 61 & 0.016393443 & 0.039340577 & 0.260805861 & 0 & 0.13761766 \\
\hline Zastów & 0.019047619 & 62 & 0.016129032 & 0.038279256 & 0.247252747 & 0 & 0.035227552 \\
\hline Baranówka & 0.019047619 & 63 & 0.015873016 & 0.037247251 & 0.233333333 & 0 & 0.018872085 \\
\hline Łuczyce & 0.019047619 & 64 & 0.015625 & 0.036244391 & 0.219047619 & 0 & 0.016535259 \\
\hline Goszcza & 0.019047619 & 65 & 0.015384615 & 0.035270406 & 0.204395604 & 0 & 0.016244752 \\
\hline Niedźwiedź & 0.019047619 & 66 & 0.015151515 & 0.034324943 & 0.189377289 & 0 & 0.016213672 \\
\hline Słomniki Miasto & 0.019047619 & 67 & 0.014925373 & 0.033407572 & 0.173992674 & 0 & 0.016210811 \\
\hline Stomniki & 0.019047619 & 68 & 0.014705882 & 0.032517807 & 0.158241758 & 0 & 0.016210583 \\
\hline Smroków & 0.019047619 & 69 & 0.014492754 & 0.03165511 & 0.142124542 & 0 & 0.016210564 \\
\hline Szczepanowice & 0.019047619 & 70 & 0.014285714 & 0.030818902 & 0.125641026 & 0 & 0.016210529 \\
\hline Kamieńczyce & 0.019047619 & 71 & 0.014084507 & 0.030008574 & 0.108791209 & 0 & 0.016210138 \\
\hline Miechów & 0.019047619 & 72 & 0.0138888889 & 0.02922349 & 0.091575092 & 0 & 0.016206433 \\
\hline Dziadówki & 0.019047619 & 73 & 0.01369863 & 0.028462998 & 0.073992674 & 0 & 0.016177795 \\
\hline Tunel & 0.019047619 & 74 & 0.013513514 & 0.027726433 & 0.056043956 & 0 & 0.016003402 \\
\hline Koztów & 0.019047619 & 75 & 0.013333333 & 0.027013121 & 0.037728938 & 0 & 0.015203531 \\
\hline Klimontów & 0.019047619 & 76 & 0.013157895 & 0.026322387 & 0.019047619 & 0 & 0.012608882 \\
\hline Sędziszów & 0.00952381 & 77 & 0.012987013 & 0.025653555 & 0 & 0 & 0.007130594 \\
\hline Kokotów & 0.038095238 & 55 & 0.018181818 & 0.044247788 & 0.460805861 & 0 & 0.270312021 \\
\hline Węgrzyce Wielkie & 0.038095238 & 54 & 0.018518519 & 0.044814341 & 0.466300366 & 0 & 0.1809432 \\
\hline Podłęże & 0.038095238 & 53 & 0.018867925 & 0.045356371 & 0.471428571 & 0 & 0.151554099 \\
\hline Staniątki & 0.038095238 & 52 & 0.019230769 & 0.04587156 & 0.476190476 & 0 & 0.143026726 \\
\hline Szarów & 0.038095238 & 51 & 0.019607843 & 0.046357616 & 0.480586081 & 0 & 0.140887727 \\
\hline Kłaj & 0.038095238 & 50 & 0.02 & 0.046812305 & 0.484615385 & 0 & 0.140427242 \\
\hline Stanisławice & 0.038095238 & 49 & 0.020408163 & 0.047233468 & 0.488278388 & 0 & 0.140342028 \\
\hline Cikowice & 0.038095238 & 48 & 0.020833333 & 0.047619048 & 0.491575092 & 0 & 0.140328287 \\
\hline Bochnia & 0.038095238 & 47 & 0.021276596 & 0.047967108 & 0.494505495 & 0 & 0.140325494 \\
\hline Rzezawa & 0.038095238 & 46 & 0.02173913 & 0.048275862 & 0.497069597 & 0 & 0.140318981 \\
\hline
\end{tabular}




\begin{tabular}{|c|c|c|c|c|c|c|c|}
\hline City (Node) & $\begin{array}{l}\text { Normalized } \\
\text { degree }\end{array}$ & Eccentricity & Radius & $\begin{array}{l}\text { Closeness } \\
\text { centrality }\end{array}$ & $\begin{array}{c}\text { Betweeness } \\
\text { centrality }\end{array}$ & Clustering & Eigencentrality \\
\hline Jasień Brzeski & 0.038095238 & 45 & 0.022222222 & 0.048543689 & 0.499267399 & 0 & 0.140280827 \\
\hline Brzesko Okocim & 0.038095238 & 44 & 0.022727273 & 0.048769159 & 0.501098901 & 0 & 0.140083543 \\
\hline Sterkowiec & 0.038095238 & 43 & 0.023255814 & 0.048951049 & 0.502564103 & 0 & 0.139226646 \\
\hline Biadoliny & 0.038095238 & 42 & 0.023809524 & 0.049088359 & 0.503663004 & 0 & 0.136154115 \\
\hline Bogumiłowice & 0.038095238 & 41 & 0.024390244 & 0.049180328 & 0.504395604 & 0 & 0.127220392 \\
\hline Tarnów Mościce & 0.038095238 & 40 & 0.025 & 0.049226442 & 0.504761905 & 0 & 0.106507052 \\
\hline Tarnów & 0.028571429 & 39 & 0.025641026 & 0.049226442 & 0.504761905 & 0 & 0.068657598 \\
\hline Kłokowa & 0.019047619 & 39 & 0.025641026 & 0.049180328 & 0.504395604 & 0 & 0.028652789 \\
\hline Łowczówek Pleśna & 0.019047619 & 40 & 0.025 & 0.049088359 & 0.503663004 & 0 & 0.018706376 \\
\hline Łowczów & 0.019047619 & 41 & 0.024390244 & 0.048951049 & 0.502564103 & 0 & 0.016624195 \\
\hline Tuchów & 0.019047619 & 42 & 0.023809524 & 0.048769159 & 0.501098901 & 0 & 0.016267124 \\
\hline Lubaszowa & 0.019047619 & 43 & 0.023255814 & 0.048543689 & 0.499267399 & 0 & 0.016217005 \\
\hline Siedliska k, Tuchowa & 0.019047619 & 44 & 0.022727273 & 0.048275862 & 0.497069597 & 0 & 0.016211187 \\
\hline Chojnik & 0.019047619 & 45 & 0.022222222 & 0.047967108 & 0.494505495 & 0 & 0.016210654 \\
\hline Gromnik & 0.019047619 & 46 & 0.02173913 & 0.047619048 & 0.491575092 & 0 & 0.016211002 \\
\hline Bogoniowice Ciężkowice & 0.019047619 & 47 & 0.021276596 & 0.047233468 & 0.488278388 & 0 & 0.016214774 \\
\hline Pławna & 0.019047619 & 48 & 0.020833333 & 0.046812305 & 0.484615385 & 0 & 0.016244203 \\
\hline Bobowa & 0.019047619 & 49 & 0.020408163 & 0.046357616 & 0.480586081 & 0 & 0.016426145 \\
\hline Bobowa Miasto & 0.019047619 & 50 & 0.02 & 0.04587156 & 0.476190476 & 0 & 0.017284875 \\
\hline Jankowa & 0.019047619 & 51 & 0.019607843 & 0.045356371 & 0.471428571 & 0 & 0.020243408 \\
\hline Wilczyska & 0.028571429 & 52 & 0.019230769 & 0.044814341 & 0.525824176 & 0 & 0.027439155 \\
\hline Polna Szalowa & 0.019047619 & 53 & 0.018867925 & 0.043334709 & 0.204395604 & 0 & 0.020243408 \\
\hline Wola Łużańska & 0.019047619 & 54 & 0.018518519 & 0.041916168 & 0.189377289 & 0 & 0.017284875 \\
\hline Moszczenica & 0.019047619 & 55 & 0.018181818 & 0.040556199 & 0.173992674 & 0 & 0.016426145 \\
\hline Małopolska & 0.019047619 & 56 & 0.017857143 & 0.039252336 & 0.158241758 & 0 & 0.016244202 \\
\hline Gorlice & 0.019047619 & 57 & 0.01754386 & 0.038002172 & 0.142124542 & 0 & 0.016214771 \\
\hline Zagórzany & 0.019047619 & 58 & 0.017241379 & 0.036803365 & 0.125641026 & 0 & 0.016210962 \\
\hline Libusza & 0.019047619 & 59 & 0.016949153 & 0.03565365 & 0.108791209 & 0 & 0.016210176 \\
\hline Biecz & 0.019047619 & 60 & 0.016666667 & 0.034550839 & 0.091575092 & 0 & 0.016206436 \\
\hline Siepietnica & 0.019047619 & 61 & 0.016393443 & 0.033492823 & 0.073992674 & 0 & 0.016177795 \\
\hline Skołoszyn & 0.019047619 & 62 & 0.016129032 & 0.032477575 & 0.056043956 & 0 & 0.016003402 \\
\hline Przysieki & 0.019047619 & 63 & 0.015873016 & 0.03150315 & 0.037728938 & 0 & 0.015203531 \\
\hline Jasło Niegłowice & 0.019047619 & 64 & 0.015625 & 0.030567686 & 0.019047619 & 0 & 0.012608882 \\
\hline Jasto & 0.00952381 & 65 & 0.015384615 & 0.029669398 & 0 & 0 & 0.007130594 \\
\hline Stróże & 0.019047619 & 53 & 0.018867925 & 0.043768237 & 0.356043956 & 0 & 0.020243408 \\
\hline Grybów & 0.019047619 & 54 & 0.018518519 & 0.042735043 & 0.345421245 & 0 & 0.017284875 \\
\hline Ptaszkowa & 0.019047619 & 55 & 0.018181818 & 0.041716329 & 0.334432234 & 0 & 0.016426145 \\
\hline Mszalnica & 0.019047619 & 56 & 0.017857143 & 0.040713455 & 0.323076923 & 0 & 0.016244203 \\
\hline Kamionka Wielka & 0.019047619 & 57 & 0.01754386 & 0.039727582 & 0.311355311 & 0 & 0.016214774 \\
\hline Nowy Sącz Jamnica & 0.019047619 & 58 & 0.017241379 & 0.03875969 & 0.299267399 & 0 & 0.016210999 \\
\hline
\end{tabular}




\begin{tabular}{|l|l|c|c|c|c|c|c|}
\hline \multicolumn{1}{|c|}{ City (Node) } & $\begin{array}{c}\text { Normalized } \\
\text { degree }\end{array}$ & Eccentricity & \multicolumn{1}{|c|}{ Radius } & $\begin{array}{c}\text { Closeness } \\
\text { centrality }\end{array}$ & $\begin{array}{c}\text { Betweeness } \\
\text { centrality }\end{array}$ & Clustering & Eigencentrality \\
\hline Nowy Sącz & 0.019047619 & 59 & 0.016949153 & 0.037810587 & 0.286813187 & 0 & 0.016210603 \\
\hline Nowy Sącz Biegonice & 0.019047619 & 60 & 0.016666667 & 0.036880927 & 0.273992674 & 0 & 0.016210569 \\
\hline Stary Sącz & 0.019047619 & 61 & 0.016393443 & 0.035971223 & 0.260805861 & 0 & 0.016210566 \\
\hline Barcice & 0.019047619 & 62 & 0.016129032 & 0.035081858 & 0.247252747 & 0 & 0.016210566 \\
\hline Rytro & 0.019047619 & 63 & 0.015873016 & 0.034213099 & 0.233333333 & 0 & 0.016210566 \\
\hline Młodów & 0.019047619 & 64 & 0.015625 & 0.03336511 & 0.219047619 & 0 & 0.016210566 \\
\hline Piwniczna Zdrój & 0.019047619 & 65 & 0.015384615 & 0.032537961 & 0.204395604 & 0 & 0.016210566 \\
\hline Piwniczna & 0.019047619 & 66 & 0.015151515 & 0.031731641 & 0.189377289 & 0 & 0.016210566 \\
\hline Łomnica Zdrój & 0.019047619 & 67 & 0.014925373 & 0.030946065 & 0.173992674 & 0 & 0.016210566 \\
\hline Wierchomla Wielka & 0.019047619 & 68 & 0.014705882 & 0.030181087 & 0.158241758 & 0 & 0.016210566 \\
\hline Zubrzyk & 0.019047619 & 69 & 0.014492754 & 0.029436501 & 0.142124542 & 0 & 0.016210563 \\
\hline Żegiestów & 0.019047619 & 70 & 0.014285714 & 0.028712059 & 0.125641026 & 0 & 0.016210529 \\
\hline Żegiestów Zdrój & 0.019047619 & 71 & 0.014084507 & 0.028007469 & 0.108791209 & 0 & 0.016210138 \\
\hline Andrzejówka & 0.019047619 & 72 & 0.013888889 & 0.027322404 & 0.091575092 & 0 & 0.016206433 \\
\hline Milik & 0.019047619 & 73 & 0.01369863 & 0.026656512 & 0.073992674 & 0 & 0.016177795 \\
\hline Muszyna & 0.019047619 & 74 & 0.013513514 & 0.026009413 & 0.056043956 & 0 & 0.016003402 \\
\hline Muszyna Zdrój & 0.019047619 & 75 & 0.013333333 & 0.025380711 & 0.037728938 & 0 & 0.015203531 \\
\hline Powroźnik & 0.019047619 & 76 & 0.013157895 & 0.024769993 & 0.019047619 & 0 & 0.012608882 \\
\hline Krynica Zdrój & 0.00952381 & 77 & 0.012987013 & 0.024176836 & 0 & 0 & 0.007130594 \\
\hline
\end{tabular}

Source: Own elaboration using Gephi software.

The figures show the distribution of sample parameters of individual network nodes. The larger the node size and the darker the colour, the larger the parameter value for the node is. 


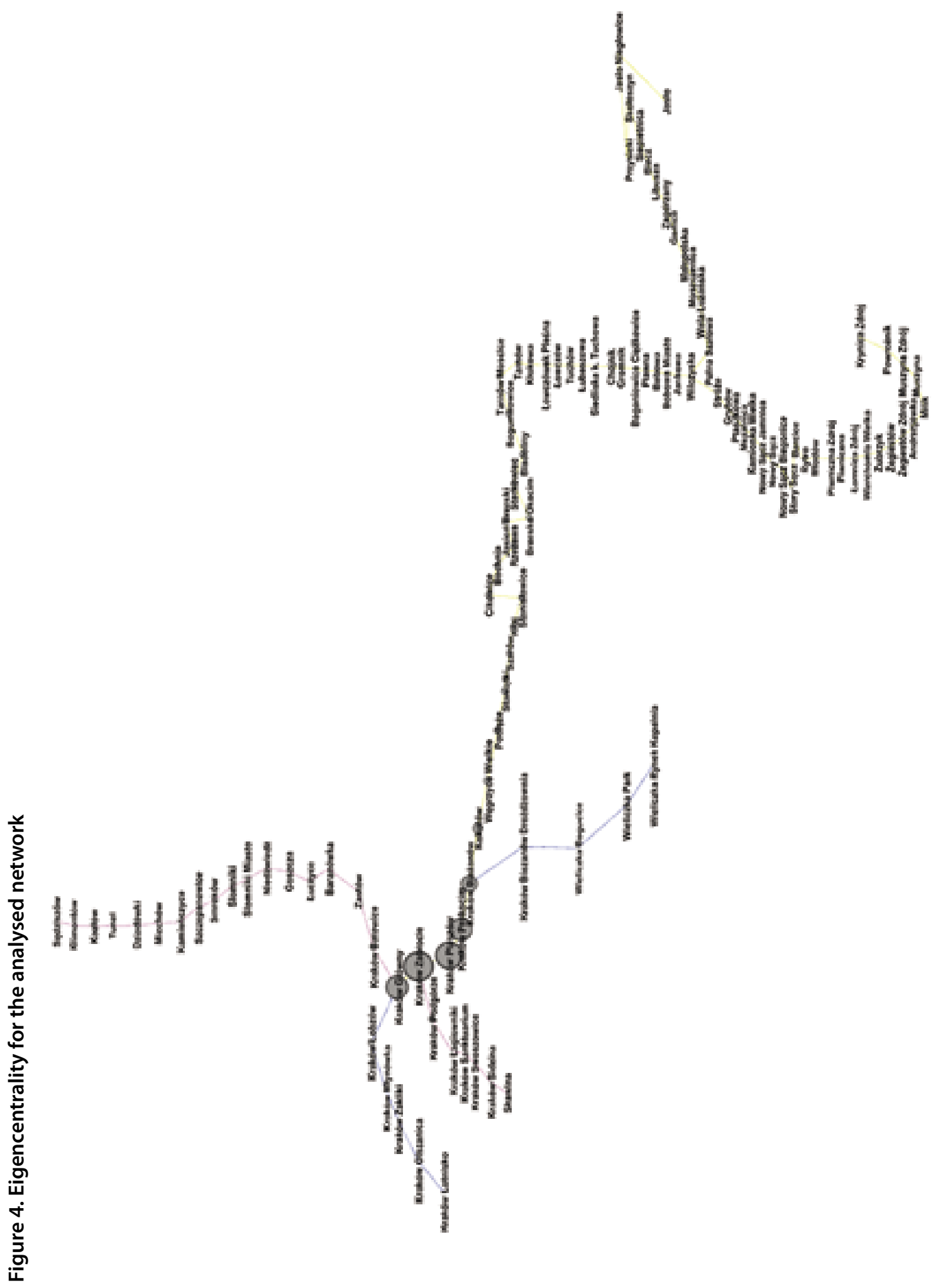




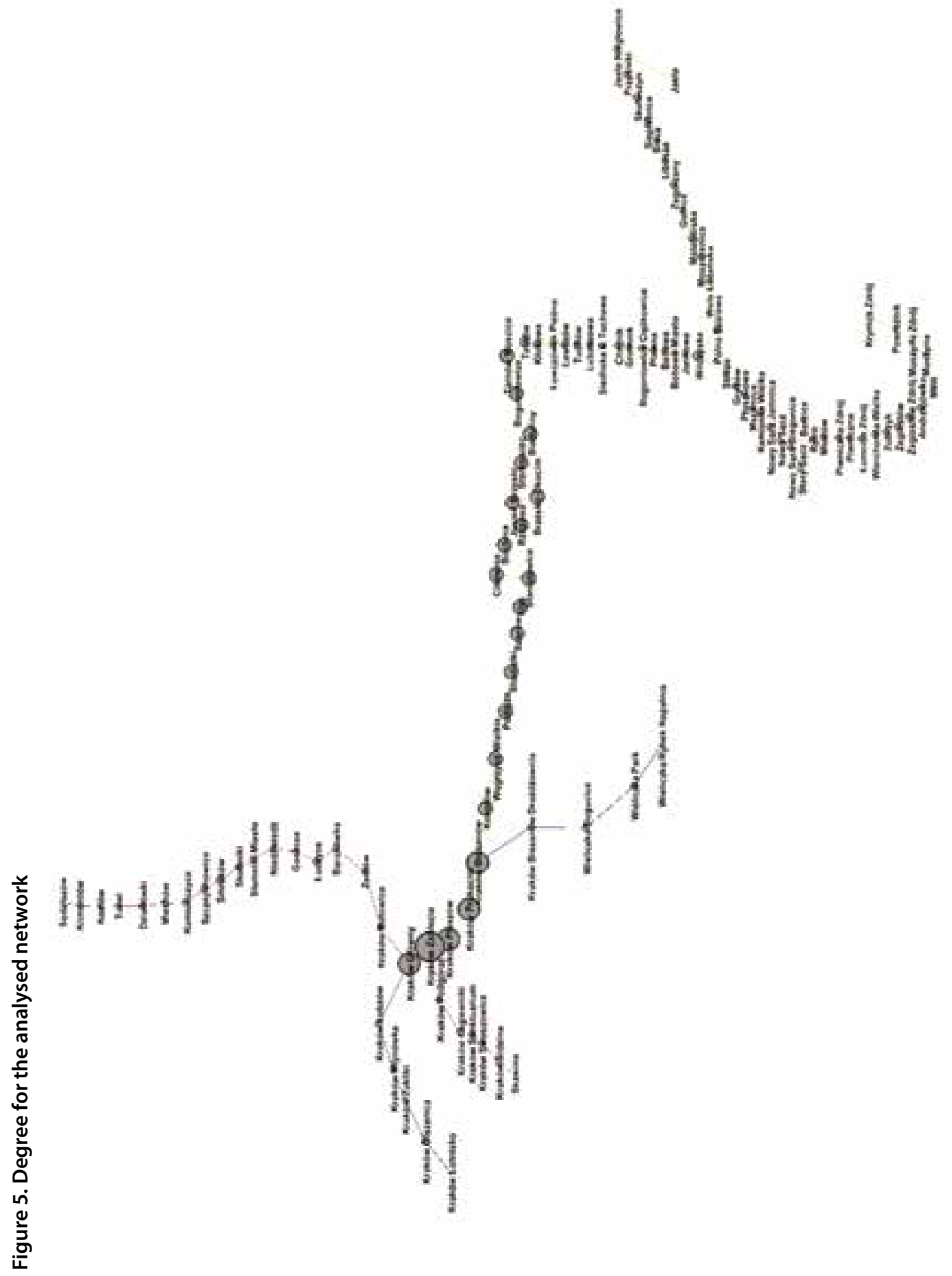


For the analysed network, its parameters as a whole were also calculated and presented in Table 3.

Table 3. Parameters of the analyzed network as a whole

\begin{tabular}{|c|c|c|c|c|}
\hline Average Clustering Coefficent & Average path length & Diameter & Radius of a network & Average nodes degree \\
\hline 0 & 27.01437556 & 77 & 39 & 2.471698113 \\
\hline
\end{tabular}

Source: the author's own elaboration based on table 2.

As presented in tables 2 and 3 and in Figures 4 and 5, the Kraków Główny, Kraków Zabłocie and Kraków Płaszów stations are the main and most important nodes in the analysed network.

\section{Modification of the connection network of Koleje Małopolskie}

Next, it was proposed to modify the network to improve its parameters. As part of the analyses, 2 modifications were proposed:

a) modification 1: added connections between Kraków Batowice and Węgrzyce Wielkie nodes, intermediate stations between them were also added (Dłubnia, Kraków Sambud, Kraków Lubocza, Kraków Nowa Huta, Kraków Nowa Huta Północ, Kraków Kościelniki, Przylasek Rusiecki, Podgrabie Wisła, Podgrabie Rudzice);

b) modification 2: connections between Kraków Łobzów and Kraków Zabłocie nodes have been added (with intermediate stations Kraków Olsza and Kraków Dąbie);

The proposed modifications are feasible because the railway infrastructure currently has a proper infrastructure which is managed by PKP PLK S.A.

Table 4 presents the results of the calculations for the connection network with the introduced modification 1.

Table 4. The parameters of individual network nodes (stations) for the analysed network connections of Koleje Małopolskie after the 1 modification.

\begin{tabular}{|l|c|c|c|c|c|c|c|}
\hline \multicolumn{1}{|c|}{ City (Node) } & $\begin{array}{c}\text { Normalized } \\
\text { degree }\end{array}$ & Eccentricity & \multicolumn{1}{c|}{ Radius } & $\begin{array}{c}\text { Closeness } \\
\text { centrality }\end{array}$ & $\begin{array}{c}\text { Betweeness } \\
\text { centrality }\end{array}$ & Clustering & Eigencentrality \\
\hline Kraków Lotnisko & 0.008695652 & 65 & 0.01538462 & 0.035870243 & 0 & 0 & 0.00713439 \\
\hline Kraków Zakliki & 0.017391304 & 63 & 0.01587302 & 0.038590604 & 0.034477498 & 0 & 0.017808284 \\
\hline Kraków Olszanica & 0.017391304 & 64 & 0.015625 & 0.037192755 & 0.017391304 & 0 & 0.012880837 \\
\hline Kraków Młynówka & 0.017391304 & 62 & 0.01612903 & 0.040069686 & 0.051258581 & 0 & 0.035043374 \\
\hline Kraków Łobzów & 0.017391304 & 61 & 0.01639344 & 0.041636495 & 0.067734554 & 0 & 0.138132514 \\
\hline Kraków Główny & 0.052173913 & 60 & 0.01666667 & 0.043298193 & 0.336668748 & 0 & 0.739029869 \\
\hline Kraków Płaszów & 0.052173913 & 58 & 0.01724138 & 0.044992175 & 0.380488144 & 0 & 0.897166719 \\
\hline Kraków Prokocim & 0.052173913 & 57 & 0.01754386 & 0.045780255 & 0.387200577 & 0 & 0.742016395 \\
\hline Kraków Bieżanów & 0.052173913 & 56 & 0.01785714 & 0.046596434 & 0.439592426 & 0 & 0.521818157 \\
\hline
\end{tabular}




\begin{tabular}{|c|c|c|c|c|c|c|c|}
\hline City (Node) & $\begin{array}{l}\text { Normalized } \\
\text { degree }\end{array}$ & Eccentricity & Radius & $\begin{array}{l}\text { Closeness } \\
\text { centrality }\end{array}$ & $\begin{array}{l}\text { Betweeness } \\
\text { centrality }\end{array}$ & Clustering & Eigencentrality \\
\hline $\begin{array}{l}\text { Kraków Bieżanów } \\
\text { Drożdżownia }\end{array}$ & 0.017391304 & 57 & 0.01754386 & 0.044642857 & 0.051258581 & 0 & 0.105111128 \\
\hline Wieliczka Bogucice & 0.017391304 & 58 & 0.01724138 & 0.042814594 & 0.034477498 & 0 & 0.029711683 \\
\hline Wieliczka Park & 0.017391304 & 59 & 0.01694915 & 0.041100786 & 0.017391304 & 0 & 0.014671765 \\
\hline Wieliczka Rynek Kopalnia & 0.008695652 & 60 & 0.01666667 & 0.039491758 & 0 & 0 & 0.007363903 \\
\hline Kraków Zabłocie & 0.069565217 & 59 & 0.01694915 & 0.044230769 & 0.400244055 & 0 & 1 \\
\hline Skawina & 0.008695652 & 65 & 0.01538462 & 0.035341119 & 0 & 0 & 0.007104748 \\
\hline Kraków Sidzina & 0.017391304 & 64 & 0.015625 & 0.036624204 & 0.017391304 & 0 & 0.012602706 \\
\hline Kraków Swoszowice & 0.017391304 & 63 & 0.01587302 & 0.037978864 & 0.034477498 & 0 & 0.015582509 \\
\hline Kraków Sanktuarium & 0.017391304 & 62 & 0.01612903 & 0.039410555 & 0.051258581 & 0 & 0.019575033 \\
\hline Kraków Łagiewniki & 0.017391304 & 61 & 0.01639344 & 0.040925267 & 0.067734554 & 0 & 0.042095676 \\
\hline Kraków Podgórze & 0.017391304 & 60 & 0.01666667 & 0.042529586 & 0.083905416 & 0 & 0.18201309 \\
\hline Kraków Batowice & 0.026086957 & 61 & 0.01639344 & 0.042248347 & 0.280383945 & 0 & 0.150939079 \\
\hline Zastów & 0.017391304 & 62 & 0.01612903 & 0.040983607 & 0.228832952 & 0 & 0.039367297 \\
\hline Baranówka & 0.017391304 & 63 & 0.01587302 & 0.039764869 & 0.215713196 & 0 & 0.019875052 \\
\hline Łuczyce & 0.017391304 & 64 & 0.015625 & 0.038590604 & 0.20228833 & 0 & 0.016674272 \\
\hline Goszcza & 0.017391304 & 65 & 0.01538462 & 0.037459283 & 0.188558352 & 0 & 0.016203739 \\
\hline Niedźwiedź & 0.017391304 & 66 & 0.01515152 & 0.036369386 & 0.174523265 & 0 & 0.016143731 \\
\hline Słomniki Miasto & 0.017391304 & 67 & 0.01492537 & 0.03531941 & 0.160183066 & 0 & 0.016137172 \\
\hline Stomniki & 0.017391304 & 68 & 0.01470588 & 0.034307876 & 0.145537757 & 0 & 0.016136558 \\
\hline Smroków & 0.017391304 & 69 & 0.01449275 & 0.033333333 & 0.130587338 & 0 & 0.016136506 \\
\hline Szczepanowice & 0.017391304 & 70 & 0.01428571 & 0.032394366 & 0.115331808 & 0 & 0.016136468 \\
\hline Kamieńczyce & 0.017391304 & 71 & 0.01408451 & 0.031489595 & 0.099771167 & 0 & 0.016136084 \\
\hline Miechów & 0.017391304 & 72 & 0.01388889 & 0.030617678 & 0.083905416 & 0 & 0.016132426 \\
\hline Dziadówki & 0.017391304 & 73 & 0.01369863 & 0.029777317 & 0.067734554 & 0 & 0.016104092 \\
\hline Tunel & 0.017391304 & 74 & 0.01351351 & 0.028967254 & 0.051258581 & 0 & 0.015931199 \\
\hline Koztów & 0.017391304 & 75 & 0.01333333 & 0.028186275 & 0.034477498 & 0 & 0.015136737 \\
\hline Klimontów & 0.017391304 & 76 & 0.01315789 & 0.027433206 & 0.017391304 & 0 & 0.012555618 \\
\hline Sędziszów & 0.008695652 & 77 & 0.01298701 & 0.026706921 & 0 & 0 & 0.007100479 \\
\hline Kokotów & 0.034782609 & 55 & 0.01818182 & 0.047286184 & 0.42299254 & 0 & 0.283343853 \\
\hline Węgrzyce Wielkie & 0.043478261 & 54 & 0.01851852 & 0.047996661 & 0.510126975 & 0 & 0.206924503 \\
\hline Podłęże & 0.034782609 & 53 & 0.01886792 & 0.048359966 & 0.493363844 & 0 & 0.165449498 \\
\hline Staniątki & 0.034782609 & 52 & 0.01923077 & 0.048687553 & 0.495804729 & 0 & 0.148665856 \\
\hline Szarów & 0.034782609 & 51 & 0.01960784 & 0.048977853 & 0.497940503 & 0 & 0.142231994 \\
\hline Kłaj & 0.034782609 & 50 & 0.02 & 0.049229452 & 0.499771167 & 0 & 0.140071171 \\
\hline Stanisławice & 0.034782609 & 49 & 0.02040816 & 0.049441101 & 0.50129672 & 0 & 0.139459953 \\
\hline Cikowice & 0.034782609 & 48 & 0.02083333 & 0.049611734 & 0.502517162 & 0 & 0.139315411 \\
\hline Bochnia & 0.034782609 & 47 & 0.0212766 & 0.049740484 & 0.503432494 & 0 & 0.139285836 \\
\hline Rzezawa & 0.034782609 & 46 & 0.02173913 & 0.04982669 & 0.504042715 & 0 & 0.139274821 \\
\hline Jasień Brzeski & 0.034782609 & 45 & 0.02222222 & 0.049869905 & 0.504347826 & 0 & 0.139236603 \\
\hline Brzesko Okocim & 0.034782609 & 44 & 0.02272727 & 0.049869905 & 0.504347826 & 0 & 0.139042042 \\
\hline
\end{tabular}




\begin{tabular}{|c|c|c|c|c|c|c|c|}
\hline City (Node) & $\begin{array}{l}\text { Normalized } \\
\text { degree }\end{array}$ & Eccentricity & Radius & $\begin{array}{l}\text { Closeness } \\
\text { centrality }\end{array}$ & $\begin{array}{c}\text { Betweeness } \\
\text { centrality }\end{array}$ & Clustering & Eigencentrality \\
\hline Sterkowiec & 0.034782609 & 43 & 0.02325581 & 0.04982669 & 0.504042715 & 0 & 0.138195769 \\
\hline Biadoliny & 0.034782609 & 42 & 0.02380952 & 0.049740484 & 0.503432494 & 0 & 0.13515627 \\
\hline Bogumiłowice & 0.034782609 & 41 & 0.02439024 & 0.049611734 & 0.502517162 & 0 & 0.126305838 \\
\hline Tarnów Mościce & 0.034782609 & 40 & 0.025 & 0.049441101 & 0.50129672 & 0 & 0.105762186 \\
\hline Tarnów & 0.026086957 & 39 & 0.02564103 & 0.049229452 & 0.499771167 & 0 & 0.068193054 \\
\hline Kłokowa & 0.017391304 & 39 & 0.02564103 & 0.048977853 & 0.497940503 & 0 & 0.02847844 \\
\hline Łowczówek Pleśna & 0.017391304 & 40 & 0.025 & 0.048687553 & 0.495804729 & 0 & 0.018609605 \\
\hline Łowczów & 0.017391304 & 41 & 0.02439024 & 0.048359966 & 0.493363844 & 0 & 0.016545792 \\
\hline Tuchów & 0.017391304 & 42 & 0.02380952 & 0.047996661 & 0.490617849 & 0 & 0.016192375 \\
\hline Lubaszowa & 0.017391304 & 43 & 0.02325581 & 0.047599338 & 0.487566743 & 0 & 0.016142854 \\
\hline Siedliska k, Tuchowa & 0.017391304 & 44 & 0.02272727 & 0.047169811 & 0.484210526 & 0 & 0.016137116 \\
\hline Chojnik & 0.017391304 & 45 & 0.02222222 & 0.046709992 & 0.480549199 & 0 & 0.016136591 \\
\hline Gromnik & 0.017391304 & 46 & 0.02173913 & 0.046221865 & 0.476582761 & 0 & 0.016136935 \\
\hline Bogoniowice Ciężkowice & 0.017391304 & 47 & 0.0212766 & 0.045707472 & 0.472311213 & 0 & 0.016140658 \\
\hline Plawna & 0.017391304 & 48 & 0.02083333 & 0.045168892 & 0.467734554 & 0 & 0.016169771 \\
\hline Bobowa & 0.017391304 & 49 & 0.02040816 & 0.044608223 & 0.462852784 & 0 & 0.016350116 \\
\hline Bobowa Miasto & 0.017391304 & 50 & 0.02 & 0.044027565 & 0.457665904 & 0 & 0.017202804 \\
\hline Jankowa & 0.017391304 & 51 & 0.01960784 & 0.043429003 & 0.452173913 & 0 & 0.020144613 \\
\hline Wilczyska & 0.026086957 & 52 & 0.01923077 & 0.042814594 & 0.495957285 & 0 & 0.027305129 \\
\hline Polna Szalowa & 0.017391304 & 53 & 0.01886792 & 0.041426513 & 0.188558352 & 0 & 0.020144613 \\
\hline Wola Łużańska & 0.017391304 & 54 & 0.01851852 & 0.040097629 & 0.174523265 & 0 & 0.017202804 \\
\hline Moszczenica & 0.017391304 & 55 & 0.01818182 & 0.038825118 & 0.160183066 & 0 & 0.016350116 \\
\hline Małopolska & 0.017391304 & 56 & 0.01785714 & 0.037606279 & 0.145537757 & 0 & 0.01616977 \\
\hline Gorlice & 0.017391304 & 57 & 0.01754386 & 0.03643853 & 0.130587338 & 0 & 0.016140655 \\
\hline Zagórzany & 0.017391304 & 58 & 0.01724138 & 0.03531941 & 0.115331808 & 0 & 0.016136895 \\
\hline Libusza & 0.017391304 & 59 & 0.01694915 & 0.034246575 & 0.099771167 & 0 & 0.01613612 \\
\hline Biecz & 0.017391304 & 60 & 0.01666667 & 0.033217793 & 0.083905416 & 0 & 0.016132428 \\
\hline Siepietnica & 0.017391304 & 61 & 0.01639344 & 0.032230942 & 0.067734554 & 0 & 0.016104092 \\
\hline Skołoszyn & 0.017391304 & 62 & 0.01612903 & 0.031284004 & 0.051258581 & 0 & 0.015931199 \\
\hline Przysieki & 0.017391304 & 63 & 0.01587302 & 0.030375066 & 0.034477498 & 0 & 0.015136737 \\
\hline Jasło Niegłowice & 0.017391304 & 64 & 0.015625 & 0.029502309 & 0.017391304 & 0 & 0.012555618 \\
\hline Jasto & 0.008695652 & 65 & 0.01538462 & 0.028664008 & 0 & 0 & 0.007100479 \\
\hline Stróże & 0.017391304 & 53 & 0.01886792 & 0.041787791 & 0.333180778 & 0 & 0.020144613 \\
\hline Grybów & 0.017391304 & 54 & 0.01851852 & 0.040780142 & 0.322807018 & 0 & 0.017202804 \\
\hline Ptaszkowa & 0.017391304 & 55 & 0.01818182 & 0.039792388 & 0.312128146 & 0 & 0.016350116 \\
\hline Mszalnica & 0.017391304 & 56 & 0.01785714 & 0.038825118 & 0.301144165 & 0 & 0.016169771 \\
\hline Kamionka Wielka & 0.017391304 & 57 & 0.01754386 & 0.037878788 & 0.289855072 & 0 & 0.016140657 \\
\hline Nowy Sącz Jamnica & 0.017391304 & 58 & 0.01724138 & 0.036953728 & 0.27826087 & 0 & 0.016136931 \\
\hline Nowy Sącz & 0.017391304 & 59 & 0.01694915 & 0.036050157 & 0.266361556 & 0 & 0.016136541 \\
\hline Nowy Sącz Biegonice & 0.017391304 & 60 & 0.01666667 & 0.035168196 & 0.254157132 & 0 & 0.016136507 \\
\hline
\end{tabular}




\begin{tabular}{|c|c|c|c|c|c|c|c|}
\hline City (Node) & $\begin{array}{l}\text { Normalized } \\
\text { degree }\end{array}$ & Eccentricity & Radius & $\begin{array}{l}\text { Closeness } \\
\text { centrality }\end{array}$ & $\begin{array}{c}\text { Betweeness } \\
\text { centrality }\end{array}$ & Clustering & Eigencentrality \\
\hline Stary Sącz & 0.017391304 & 61 & 0.01639344 & 0.034307876 & 0.241647597 & 0 & 0.016136505 \\
\hline Barcice & 0.017391304 & 62 & 0.01612903 & 0.03346915 & 0.228832952 & 0 & 0.016136505 \\
\hline Rytro & 0.017391304 & 63 & 0.01587302 & 0.032651902 & 0.215713196 & 0 & 0.016136505 \\
\hline Młodów & 0.017391304 & 64 & 0.015625 & 0.031855956 & 0.20228833 & 0 & 0.016136505 \\
\hline Piwniczna Zdrój & 0.017391304 & 65 & 0.01538462 & 0.031081081 & 0.188558352 & 0 & 0.016136505 \\
\hline Piwniczna & 0.017391304 & 66 & 0.01515152 & 0.030327004 & 0.174523265 & 0 & 0.016136505 \\
\hline Łomnica Zdrój & 0.017391304 & 67 & 0.01492537 & 0.029593412 & 0.160183066 & 0 & 0.016136505 \\
\hline Wierchomla Wielka & 0.017391304 & 68 & 0.01470588 & 0.02887996 & 0.145537757 & 0 & 0.016136505 \\
\hline Zubrzyk & 0.017391304 & 69 & 0.01449275 & 0.028186275 & 0.130587338 & 0 & 0.016136502 \\
\hline Żegiestów & 0.017391304 & 70 & 0.01428571 & 0.027511962 & 0.115331808 & 0 & 0.016136468 \\
\hline Żegiestów Zdrój & 0.017391304 & 71 & 0.01408451 & 0.026856609 & 0.099771167 & 0 & 0.016136084 \\
\hline Andrzejówka & 0.017391304 & 72 & 0.01388889 & 0.02621979 & 0.083905416 & 0 & 0.016132426 \\
\hline Milik & 0.017391304 & 73 & 0.01369863 & 0.025601069 & 0.067734554 & 0 & 0.016104092 \\
\hline Muszyna & 0.017391304 & 74 & 0.01351351 & 0.025 & 0.051258581 & 0 & 0.015931199 \\
\hline Muszyna Zdrój & 0.017391304 & 75 & 0.01333333 & 0.024416136 & 0.034477498 & 0 & 0.015136737 \\
\hline Powroźnik & 0.017391304 & 76 & 0.01315789 & 0.023849025 & 0.017391304 & 0 & 0.012555618 \\
\hline Krynica Zdrój & 0.008695652 & 77 & 0.01298701 & 0.023298217 & 0 & 0 & 0.007100479 \\
\hline Rudzice & 0.017391304 & 55 & 0.01818182 & 0.046110666 & 0.086200102 & 0 & 0.055746749 \\
\hline Podgrabie & 0.017391304 & 56 & 0.01785714 & 0.044367284 & 0.071103469 & 0 & 0.023521969 \\
\hline Podgrabie Wisła & 0.017391304 & 57 & 0.01754386 & 0.043265613 & 0.058449483 & 0 & 0.017317161 \\
\hline Przylasek Rusiecki & 0.017391304 & 58 & 0.01724138 & 0.042372881 & 0.049031307 & 0 & 0.016295364 \\
\hline Kraków Kościelniki & 0.017391304 & 59 & 0.01694915 & 0.041696882 & 0.041327264 & 0 & 0.016155131 \\
\hline $\begin{array}{l}\text { Kraków Nowa Huta } \\
\text { Północ }\end{array}$ & 0.017391304 & 60 & 0.01666667 & 0.041042113 & 0.034614831 & 0 & 0.016145452 \\
\hline Kraków Nowa Huta & 0.017391304 & 61 & 0.01639344 & 0.04040759 & 0.028599793 & 0 & 0.01620388 \\
\hline Kraków Lubocza & 0.017391304 & 62 & 0.01612903 & 0.039902845 & 0.023231716 & 0 & 0.016674282 \\
\hline Kraków Sambud & 0.017391304 & 63 & 0.01587302 & 0.039410555 & 0.028088128 & 0 & 0.019875053 \\
\hline Dłubnia & 0.017391304 & 62 & 0.01612903 & 0.040780142 & 0.043166793 & 0 & 0.039367297 \\
\hline
\end{tabular}

Source: the author's own elaboration using the Gephi software.

Figures 6 and 7 present the distribution of exemplary parameters of individual network nodes for the network after the 1 modification. As in the previous drawings, the larger the node size and the darker the colour, the parameter value for a given node is larger. 


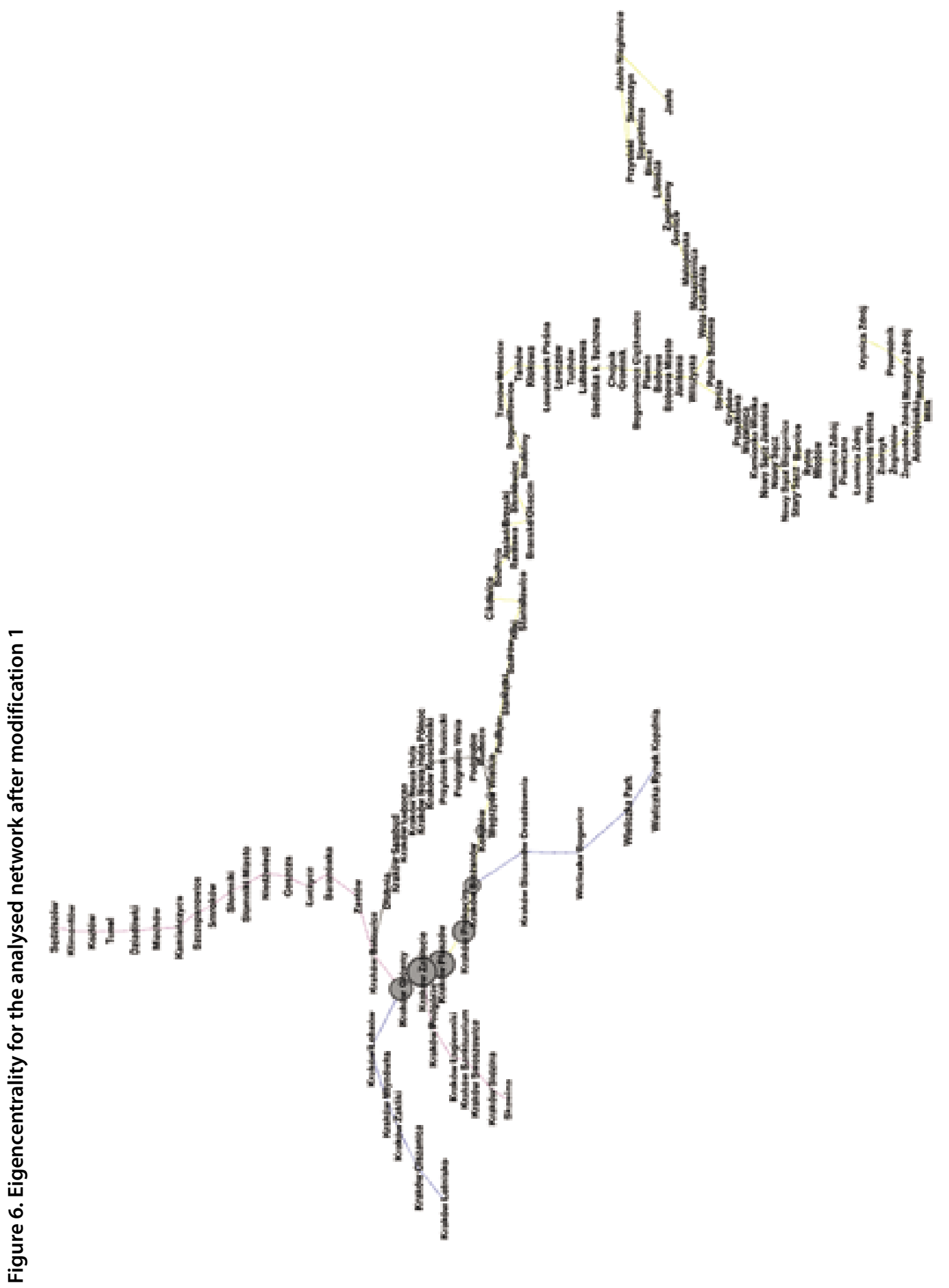




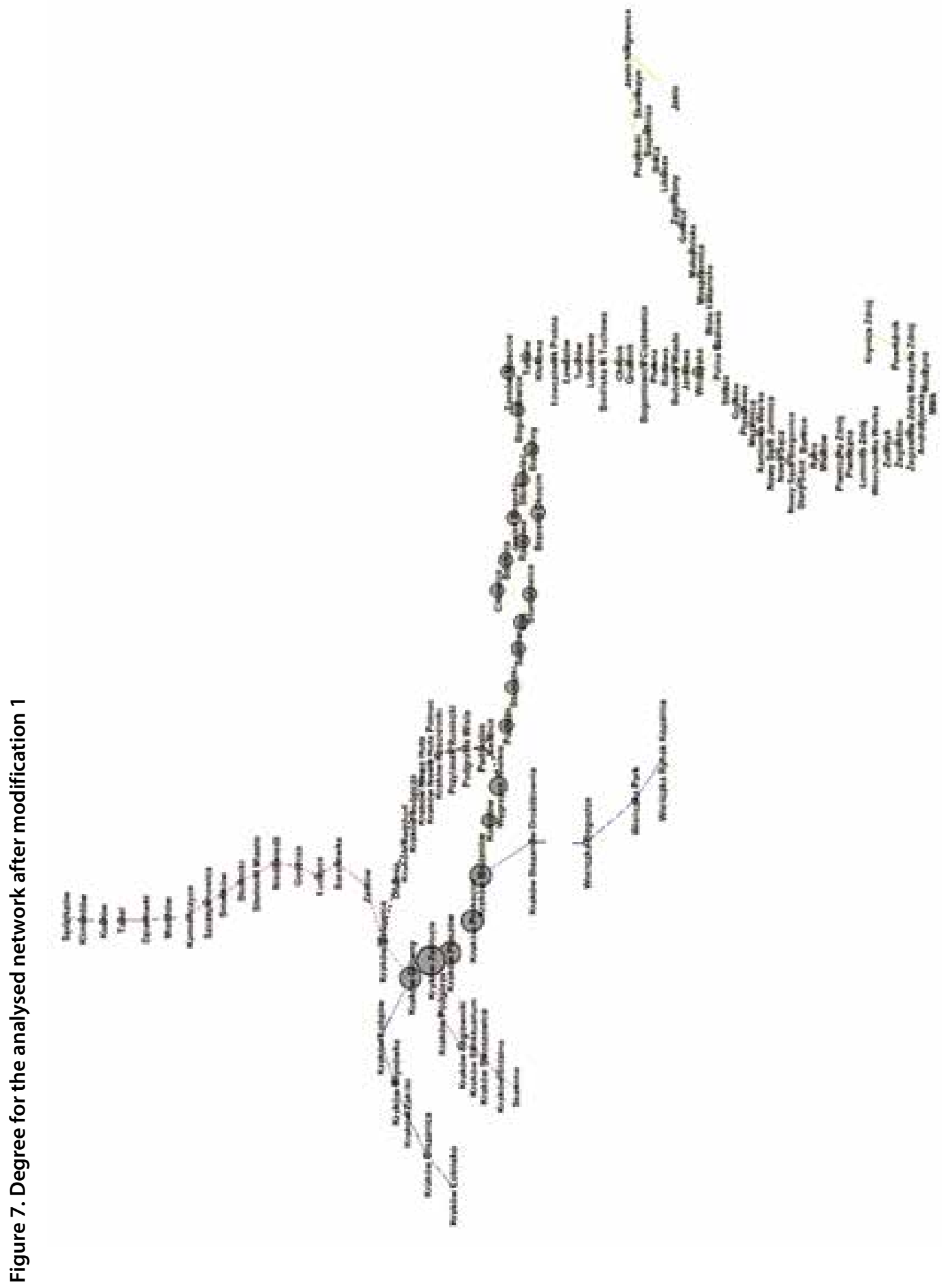


Then indicators for the entire network were calculated. Their values are presented in Table 5.

Table 5. Parameters of the analyzed network after 1 modification as a whole

\begin{tabular}{|c|c|c|c|c|}
\hline Average Clustering Coefficent & Average path length & Diameter & Radius of a network & Average nodes degree \\
\hline 0 & 26.6347826 & 77 & 39 & 2.448276 \\
\hline
\end{tabular}

Source: the author's own elaboration based on table 4.

As shown in Tables 4 and 5 and Figures 6 and 7 in the network after the modification, the Kraków Główny, Kraków Zabłocie and Kraków Płaszów stations still play the main role but the average path length has improved, which is a good result of the proposed modification, but unfortunately the average degree of the network node has deteriorated.

Next, the parameters for the network were calculated after the addition of modification 2, the results of the calculations of individual node parameters are presented in Table 6.

Table 6. Parameters of individual network nodes (stations) for the analysed network of Koleje Małopolskie connections after modification 2.

\begin{tabular}{|c|c|c|c|c|c|c|c|}
\hline City (Node) & $\begin{array}{l}\text { Normalized } \\
\text { degree }\end{array}$ & Eccentricity & Radius & $\begin{array}{l}\text { Closeness } \\
\text { centrality }\end{array}$ & $\begin{array}{c}\text { Betweeness } \\
\text { centrality }\end{array}$ & Clustering & Eigencentrality \\
\hline Kraków Lotnisko & 0.008547009 & 65 & 0.01538462 & 0.036369288 & 0 & 0 & 0.006813712 \\
\hline Kraków Zakliki & 0.017094017 & 63 & 0.01587302 & 0.039169736 & 0.03389331 & 0 & 0.017925986 \\
\hline Kraków Olszanica & 0.017094017 & 64 & 0.015625 & 0.037729765 & 0.017094017 & 0 & 0.012442416 \\
\hline Kraków Młynówka & 0.017094017 & 62 & 0.01612903 & 0.040695652 & 0.050397878 & 0 & 0.037877347 \\
\hline Kraków Łobzów & 0.025641026 & 61 & 0.01639344 & 0.042314647 & 0.070291777 & 0 & 0.150873621 \\
\hline Kraków Główny & 0.051282051 & 60 & 0.01666667 & 0.043984962 & 0.332139604 & 0 & 0.734037724 \\
\hline Kraków Płaszów & 0.051282051 & 58 & 0.01724138 & 0.045685279 & 0.390666677 & 0 & 0.862267729 \\
\hline Kraków Prokocim & 0.051282051 & 57 & 0.01754386 & 0.046447003 & 0.39685589 & 0 & 0.693278835 \\
\hline Kraków Bieżanów & 0.051282051 & 56 & 0.01785714 & 0.047234558 & 0.447169561 & 0 & 0.479516346 \\
\hline $\begin{array}{l}\text { Kraków Bieżanów } \\
\text { Drożdżownia }\end{array}$ & 0.017094017 & 57 & 0.01754386 & 0.045226131 & 0.050397878 & 0 & 0.095942883 \\
\hline Wieliczka Bogucice & 0.017094017 & 58 & 0.01724138 & 0.043349389 & 0.03389331 & 0 & 0.027398187 \\
\hline Wieliczka Park & 0.017094017 & 59 & 0.01694915 & 0.041592606 & 0.017094017 & 0 & 0.013808996 \\
\hline Wieliczka Rynek Kopalnia & 0.008547009 & 60 & 0.01666667 & 0.039945374 & 0 & 0 & 0.006982181 \\
\hline Kraków Zabłocie & 0.076923077 & 59 & 0.01694915 & 0.044948137 & 0.415355111 & 0 & 1 \\
\hline Skawina & 0.008547009 & 65 & 0.01538462 & 0.035790762 & 0 & 0 & 0.006757824 \\
\hline Kraków Sidzina & 0.017094017 & 64 & 0.015625 & 0.037107517 & 0.017094017 & 0 & 0.011989969 \\
\hline Kraków Swoszowice & 0.017094017 & 63 & 0.01587302 & 0.038499506 & 0.03389331 & 0 & 0.01479442 \\
\hline Kraków Sanktuarium & 0.017094017 & 62 & 0.01612903 & 0.039972668 & 0.050397878 & 0 & 0.018640885 \\
\hline Kraków Łagiewniki & 0.017094017 & 61 & 0.01639344 & 0.041533546 & 0.066607722 & 0 & 0.040792232 \\
\hline Kraków Podgórze & 0.017094017 & 60 & 0.01666667 & 0.043189369 & 0.082522841 & 0 & 0.179788481 \\
\hline Kraków Batowice & 0.025641026 & 61 & 0.01639344 & 0.042888563 & 0.27747605 & 0 & 0.147357028 \\
\hline Zastów & 0.017094017 & 62 & 0.01612903 & 0.041577825 & 0.225464191 & 0 & 0.03763224 \\
\hline Baranówka & 0.017094017 & 63 & 0.01587302 & 0.040317023 & 0.212496316 & 0 & 0.018795096 \\
\hline
\end{tabular}




\begin{tabular}{|c|c|c|c|c|c|c|c|}
\hline City (Node) & $\begin{array}{l}\text { Normalized } \\
\text { degree }\end{array}$ & Eccentricity & Radius & $\begin{array}{l}\text { Closeness } \\
\text { centrality }\end{array}$ & $\begin{array}{l}\text { Betweeness } \\
\text { centrality }\end{array}$ & Clustering & Eigencentrality \\
\hline Łuczyce & 0.017094017 & 64 & 0.015625 & 0.039104278 & 0.199233716 & 0 & 0.015772479 \\
\hline Goszcza & 0.017094017 & 65 & 0.01538462 & 0.037937743 & 0.185676393 & 0 & 0.015339375 \\
\hline Niedźwiedź & 0.017094017 & 66 & 0.01515152 & 0.036815607 & 0.171824344 & 0 & 0.015285532 \\
\hline Słomniki Miasto & 0.017094017 & 67 & 0.01492537 & 0.035736103 & 0.157677571 & 0 & 0.015279787 \\
\hline Stomniki & 0.017094017 & 68 & 0.01470588 & 0.034697509 & 0.143236074 & 0 & 0.015279261 \\
\hline Smroków & 0.017094017 & 69 & 0.01449275 & 0.033698157 & 0.128499853 & 0 & 0.015279217 \\
\hline Szczepanowice & 0.017094017 & 70 & 0.01428571 & 0.03273643 & 0.113468907 & 0 & 0.015279186 \\
\hline Kamieńczyce & 0.017094017 & 71 & 0.01408451 & 0.031810767 & 0.098143236 & 0 & 0.015278861 \\
\hline Miechów & 0.017094017 & 72 & 0.01388889 & 0.030919662 & 0.082522841 & 0 & 0.015275703 \\
\hline Dziadówki & 0.017094017 & 73 & 0.01369863 & 0.030061665 & 0.066607722 & 0 & 0.01525075 \\
\hline Tunel & 0.017094017 & 74 & 0.01351351 & 0.029235382 & 0.050397878 & 0 & 0.015095255 \\
\hline Koztów & 0.017094017 & 75 & 0.01333333 & 0.028439475 & 0.03389331 & 0 & 0.014365306 \\
\hline Klimontów & 0.017094017 & 76 & 0.01315789 & 0.027672658 & 0.017094017 & 0 & 0.011945164 \\
\hline Sędziszów & 0.008547009 & 77 & 0.01298701 & 0.026933702 & 0 & 0 & 0.00675381 \\
\hline Kokotów & 0.034188034 & 55 & 0.01818182 & 0.047891936 & 0.429661124 & 0 & 0.25792823 \\
\hline Węgrzyce Wielkie & 0.042735043 & 54 & 0.01851852 & 0.04856787 & 0.513534719 & 0 & 0.188232396 \\
\hline Podłęże & 0.034188034 & 53 & 0.01886792 & 0.048892603 & 0.49602122 & 0 & 0.150760597 \\
\hline Staniątki & 0.034188034 & 52 & 0.01923077 & 0.049180328 & 0.498084291 & 0 & 0.135684846 \\
\hline Szarów & 0.034188034 & 51 & 0.019 & 0.049429658 & 0.499852638 & 0 & 0.129948551 \\
\hline Kłaj & 0.034188034 & 50 & 0.02 & 0.049639372 & 0.50132626 & 0 & 0.128044081 \\
\hline Stanisławice & 0.034188034 & 49 & 0.02040816 & 0.049808429 & 0.502505158 & 0 & 0.127513165 \\
\hline Cikowice & 0.034188034 & 48 & 0.02083333 & 0.04993598 & 0.503389331 & 0 & 0.127389626 \\
\hline Bochnia & 0.034188034 & 47 & 0.0212766 & 0.050021377 & 0.50397878 & 0 & 0.127364792 \\
\hline Rzezawa & 0.034188034 & 46 & 0.02173913 & 0.050064185 & 0.504273504 & 0 & 0.127355702 \\
\hline Jasień Brzeski & 0.034188034 & 45 & 0.02222222 & 0.050064185 & 0.504273504 & 0 & 0.127323694 \\
\hline Brzesko Okocim & 0.034188034 & 44 & 0.02272727 & 0.050021377 & 0.50397878 & 0 & 0.1271579 \\
\hline Sterkowiec & 0.034188034 & 43 & 0.02325581 & 0.04993598 & 0.503389331 & 0 & 0.126424528 \\
\hline Biadoliny & 0.034188034 & 42 & 0.02380952 & 0.049808429 & 0.502505158 & 0 & 0.123747152 \\
\hline Bogumiłowice & 0.034188034 & 41 & 0.02439024 & 0.049639372 & 0.50132626 & 0 & 0.115831956 \\
\hline Tarnów Mościce & 0.034188034 & 40 & 0.025 & 0.049429658 & 0.499852638 & 0 & 0.09721996 \\
\hline Tarnów & 0.025641026 & 39 & 0.02564103 & 0.049180328 & 0.498084291 & 0 & 0.062859123 \\
\hline Kłokowa & 0.017094017 & 39 & 0.02564103 & 0.048892603 & 0.49602122 & 0 & 0.026473535 \\
\hline Łowczówek Pleśna & 0.017094017 & 40 & 0.025 & 0.04856787 & 0.493663425 & 0 & 0.017495364 \\
\hline Łowczów & 0.017094017 & 41 & 0.02439024 & 0.048207664 & 0.491010905 & 0 & 0.015640511 \\
\hline Tuchów & 0.017094017 & 42 & 0.02380952 & 0.047813649 & 0.48806366 & 0 & 0.015327728 \\
\hline Lubaszowa & 0.017094017 & 43 & 0.02325581 & 0.047387606 & 0.484821692 & 0 & 0.015284636 \\
\hline Siedliska k, Tuchowa & 0.017094017 & 44 & 0.02272727 & 0.046931408 & 0.481284999 & 0 & 0.015279729 \\
\hline Chojnik & 0.017094017 & 45 & 0.02222222 & 0.046447003 & 0.477453581 & 0 & 0.015279288 \\
\hline Gromnik & 0.017094017 & 46 & 0.02173913 & 0.045936396 & 0.473327439 & 0 & 0.015279579 \\
\hline Bogoniowice Ciężkowice & 0.017094017 & 47 & 0.0212766 & 0.04540163 & 0.468906572 & 0 & 0.015282791 \\
\hline
\end{tabular}




\begin{tabular}{|c|c|c|c|c|c|c|c|}
\hline City (Node) & $\begin{array}{l}\text { Normalized } \\
\text { degree }\end{array}$ & Eccentricity & Radius & $\begin{array}{c}\text { Closeness } \\
\text { centrality }\end{array}$ & $\begin{array}{c}\text { Betweeness } \\
\text { centrality }\end{array}$ & Clustering & Eigencentrality \\
\hline Pławna & 0.017094017 & 48 & 0.02083333 & 0.044844768 & 0.464190981 & 0 & 0.015308403 \\
\hline Bobowa & 0.017094017 & 49 & 0.02040816 & 0.044267877 & 0.459180666 & 0 & 0.015470326 \\
\hline Bobowa Miasto & 0.017094017 & 50 & 0.02 & 0.043673012 & 0.453875626 & 0 & 0.0162515 \\
\hline Jankowa & 0.017094017 & 51 & 0.01960784 & 0.043062201 & 0.448275862 & 0 & 0.018995489 \\
\hline Wilczyska & 0.025641026 & 52 & 0.01923077 & 0.042437432 & 0.490274094 & 0 & 0.02574919 \\
\hline Polna Szalowa & 0.017094017 & 53 & 0.01886792 & 0.041067041 & 0.185676393 & 0 & 0.018995489 \\
\hline Wola Łużańska & 0.017094017 & 54 & 0.01851852 & 0.039755352 & 0.171824344 & 0 & 0.0162515 \\
\hline Moszczenica & 0.017094017 & 55 & 0.01818182 & 0.038499506 & 0.157677571 & 0 & 0.015470326 \\
\hline Małopolska & 0.017094017 & 56 & 0.01785714 & 0.03729678 & 0.143236074 & 0 & 0.015308403 \\
\hline Gorlice & 0.017094017 & 57 & 0.01754386 & 0.036144578 & 0.128499853 & 0 & 0.015282788 \\
\hline Zagórzany & 0.017094017 & 58 & 0.01724138 & 0.035040431 & 0.113468907 & 0 & 0.015279546 \\
\hline Libusza & 0.017094017 & 59 & 0.01694915 & 0.033981992 & 0.098143236 & 0 & 0.015278891 \\
\hline Biecz & 0.017094017 & 60 & 0.01666667 & 0.032967033 & 0.082522841 & 0 & 0.015275706 \\
\hline Siepietnica & 0.017094017 & 61 & 0.01639344 & 0.031993437 & 0.066607722 & 0 & 0.01525075 \\
\hline Skołoszyn & 0.017094017 & 62 & 0.01612903 & 0.031059198 & 0.050397878 & 0 & 0.015095255 \\
\hline Przysieki & 0.017094017 & 63 & 0.01587302 & 0.030162413 & 0.03389331 & 0 & 0.014365306 \\
\hline Jasło Niegłowice & 0.017094017 & 64 & 0.015625 & 0.029301277 & 0.017094017 & 0 & 0.011945164 \\
\hline Jasto & 0.008547009 & 65 & 0.01538462 & 0.028474081 & 0 & 0 & 0.00675381 \\
\hline Stróże & 0.017094017 & 53 & 0.01886792 & 0.041415929 & 0.328912467 & 0 & 0.018995489 \\
\hline Grybów & 0.017094017 & 54 & 0.01851852 & 0.040414508 & 0.318597112 & 0 & 0.0162515 \\
\hline Ptaszkowa & 0.017094017 & 55 & 0.01818182 & 0.039433771 & 0.307987032 & 0 & 0.015470326 \\
\hline Mszalnica & 0.017094017 & 56 & 0.01785714 & 0.038474186 & 0.297082228 & 0 & 0.015308403 \\
\hline Kamionka Wielka & 0.017094017 & 57 & 0.01754386 & 0.037536092 & 0.2858827 & 0 & 0.01528279 \\
\hline Nowy Sącz Jamnica & 0.017094017 & 58 & 0.01724138 & 0.036619718 & 0.274388447 & 0 & 0.015279577 \\
\hline Nowy Sącz & 0.017094017 & 59 & 0.01694915 & 0.035725191 & 0.262599469 & 0 & 0.015279247 \\
\hline Nowy Sącz Biegonice & 0.017094017 & 60 & 0.01666667 & 0.034852547 & 0.250515768 & 0 & 0.015279219 \\
\hline Stary Sącz & 0.017094017 & 61 & 0.01639344 & 0.034001744 & 0.238137342 & 0 & 0.015279216 \\
\hline Barcice & 0.017094017 & 62 & 0.01612903 & 0.033172668 & 0.225464191 & 0 & 0.015279216 \\
\hline Rytro & 0.017094017 & 63 & 0.01587302 & 0.032365145 & 0.212496316 & 0 & 0.015279216 \\
\hline Młodów & 0.017094017 & 64 & 0.015625 & 0.031578947 & 0.199233716 & 0 & 0.015279216 \\
\hline Piwniczna Zdrój & 0.017094017 & 65 & 0.01538462 & 0.0308138 & 0.185676393 & 0 & 0.015279216 \\
\hline Piwniczna & 0.017094017 & 66 & 0.01515152 & 0.030069391 & 0.171824344 & 0 & 0.015279216 \\
\hline Łomnica Zdrój & 0.017094017 & 67 & 0.01492537 & 0.029345372 & 0.157677571 & 0 & 0.015279216 \\
\hline Wierchomla Wielka & 0.017094017 & 68 & 0.01470588 & 0.028641371 & 0.143236074 & 0 & 0.015279216 \\
\hline Zubrzyk & 0.017094017 & 69 & 0.01449275 & 0.027956989 & 0.128499853 & 0 & 0.015279214 \\
\hline Żegiestów & 0.017094017 & 70 & 0.01428571 & 0.027291812 & 0.113468907 & 0 & 0.015279186 \\
\hline Żegiestów Zdrój & 0.017094017 & 71 & 0.01408451 & 0.026645411 & 0.098143236 & 0 & 0.015278861 \\
\hline Andrzejówka & 0.017094017 & 72 & 0.01388889 & 0.026017345 & 0.082522841 & 0 & 0.015275703 \\
\hline Milik & 0.017094017 & 73 & 0.01369863 & 0.025407166 & 0.066607722 & 0 & 0.01525075 \\
\hline Muszyna & 0.017094017 & 74 & 0.01351351 & 0.024814422 & 0.050397878 & 0 & 0.015095255 \\
\hline
\end{tabular}




\begin{tabular}{|l|c|c|c|c|c|c|c|}
\hline \multicolumn{1}{|c|}{ City (Node) } & $\begin{array}{c}\text { Normalized } \\
\text { degree }\end{array}$ & Eccentricity & Radius & $\begin{array}{c}\text { Closeness } \\
\text { centrality }\end{array}$ & $\begin{array}{c}\text { Betweeness } \\
\text { centrality }\end{array}$ & Clustering & Eigencentrality \\
\hline Muszyna Zdrój & 0.017094017 & 75 & 0.01333333 & 0.024238658 & 0.03389331 & 0 & 0.014365306 \\
\hline Powroźnik & 0.017094017 & 76 & 0.01315789 & 0.023679417 & 0.017094017 & 0 & 0.011945164 \\
\hline Krynica Zdrój & 0.008547009 & 77 & 0.01298701 & 0.023136247 & 0 & 0 & 0.00675381 \\
\hline Rudzice & 0.017094017 & 55 & 0.01818182 & 0.046632124 & 0.083997341 & 0 & 0.050984687 \\
\hline Podgrabie & 0.017094017 & 56 & 0.01785714 & 0.044844768 & 0.069119883 & 0 & 0.021872706 \\
\hline Podgrabie Wisła & 0.017094017 & 57 & 0.01754386 & 0.043705641 & 0.056601922 & 0 & 0.016319424 \\
\hline Przylasek Rusiecki & 0.017094017 & 58 & 0.01724138 & 0.04279444 & 0.047362248 & 0 & 0.015417038 \\
\hline Kraków Kościelniki & 0.017094017 & 59 & 0.01694915 & 0.042146974 & 0.040073082 & 0 & 0.015295114 \\
\hline $\begin{array}{l}\text { Kraków Nowa Huta } \\
\text { Północ }\end{array}$ & 0.017094017 & 60 & 0.01666667 & 0.041518808 & 0.033883868 & 0 & 0.015286975 \\
\hline Kraków Nowa Huta & 0.017094017 & 61 & 0.01639344 & 0.040909091 & 0.028368311 & 0 & 0.015339492 \\
\hline Kraków Lubocza & 0.017094017 & 62 & 0.01612903 & 0.040428473 & 0.023477691 & 0 & 0.015772487 \\
\hline Kraków Sambud & 0.017094017 & 63 & 0.01587302 & 0.039959016 & 0.028463512 & 0 & 0.018795096 \\
\hline Dłubnia & 0.017094017 & 62 & 0.01612903 & 0.041371994 & 0.043323614 & 0 & 0.03763224 \\
\hline Kraków Olsza & 0.017094017 & 61 & 0.01639344 & 0.04189044 & $7.37 E-04$ & 0 & 0.063163892 \\
\hline Kraków Dąbie & 0.017094017 & 60 & 0.01666667 & 0.043141593 & 0.012673151 & 0 & 0.183247331 \\
\hline
\end{tabular}

Source: the author's own elaboration using the Gephi software.

The figures show the distribution of the exemplary parameters of individual network nodes for the network after the $2^{\text {nd }}$ modification. As in the previous drawings, the larger the node size and the darker the colour, the parameter value for the given node is greater. 


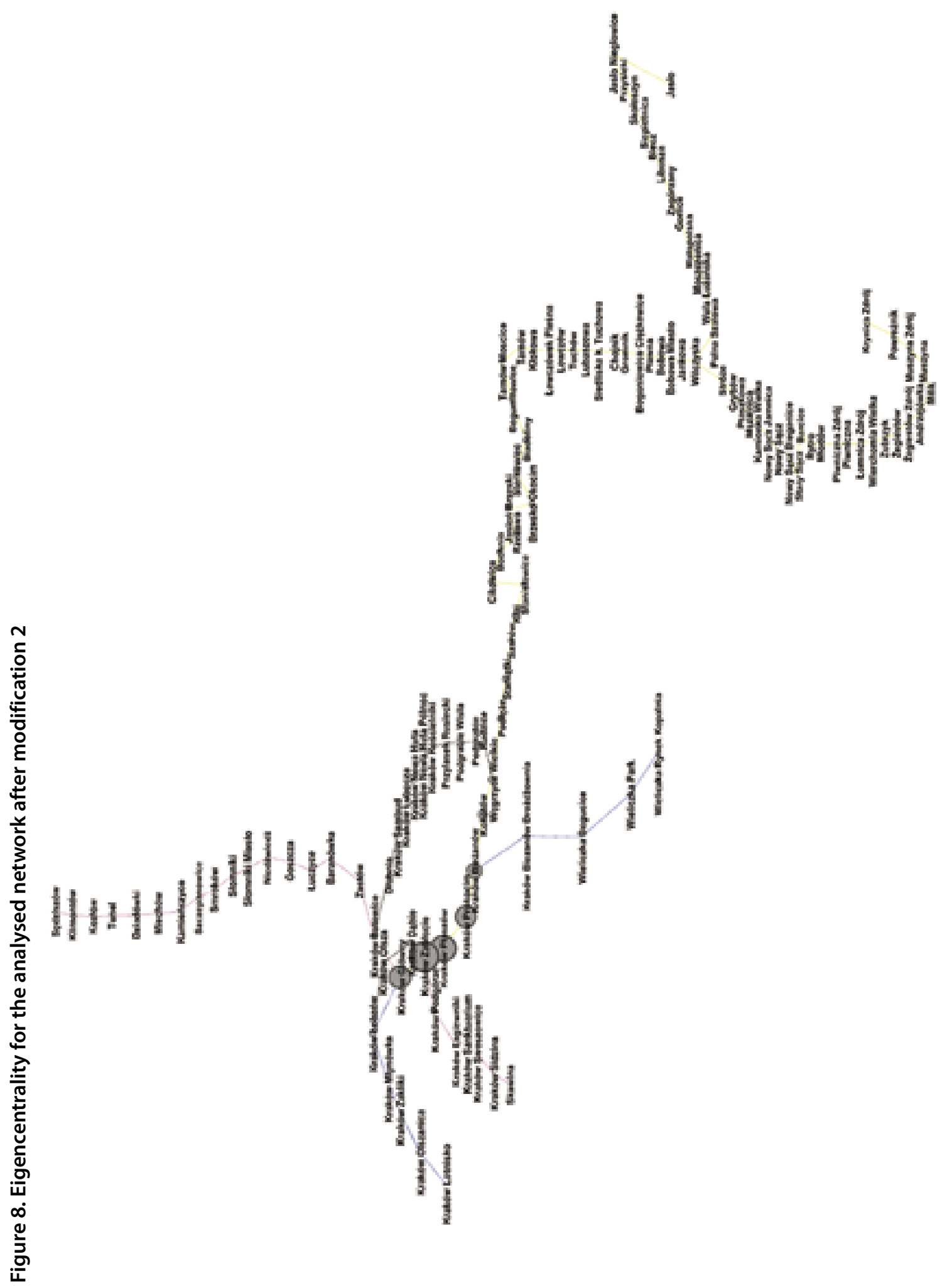




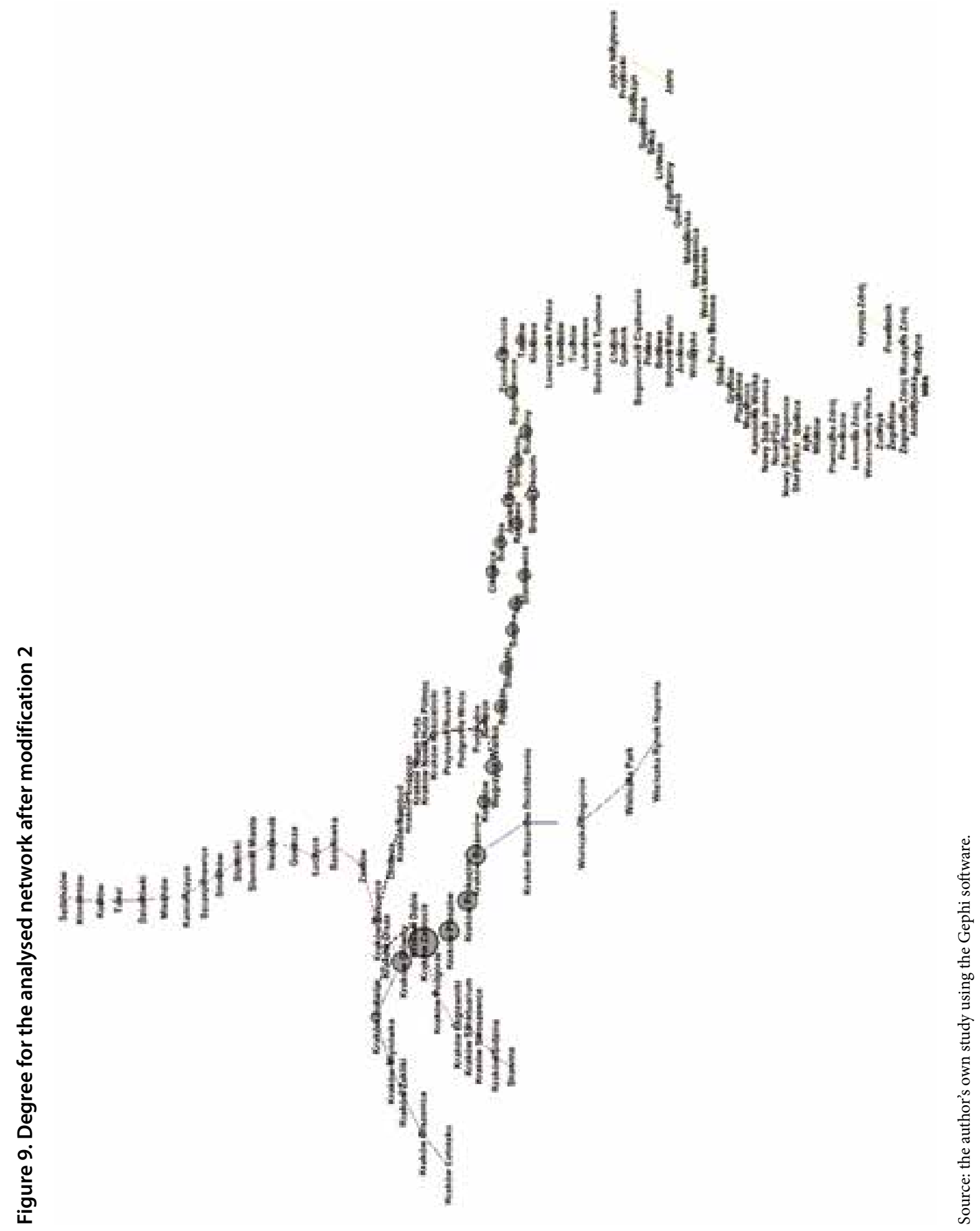


Then the indicators for the entire network were calculated. Their values are presented in Table 7.

Table 7. Parameters of the analyzed network after modification 2 as a whole

\begin{tabular}{|c|c|c|c|c|}
\hline Average Clustering Coefficent & Average path length & Diameter & Radius of a network & Average nodes degree \\
\hline 0 & 26.53310155 & 77 & 39 & 2.457627119 \\
\hline
\end{tabular}

Source: the author's own elaboration based on table 6.

As shown in Tables 6 and 7 and Figures 8 and 9, the introduced modification did not change the validity of individual network nodes but contributed to a slight improvement in the average path length.

In order to better illustrate the effect of the proposed changes in the network parameters, table 8 presents the parameters of the original network and networks with the proposed modifications.

Table 8. Comparison of the parameters of the analyzed network before and after the modifications

\begin{tabular}{|l|c|c|c|c|c|}
\cline { 2 - 5 } \multicolumn{1}{c|}{} & $\begin{array}{c}\text { Average Clustering } \\
\text { Coefficent }\end{array}$ & $\begin{array}{c}\text { Average path } \\
\text { length }\end{array}$ & Diameter & $\begin{array}{c}\text { Radius of } \\
\text { a network }\end{array}$ & $\begin{array}{c}\text { Average nodes } \\
\text { degree }\end{array}$ \\
\hline Original network & 0 & 27.01437556 & 77 & 39 & 2.471698113 \\
\hline Network after modification 1 & 0 & 26.6347826 & 77 & 39 & 2.448276 \\
\hline Network after modification 2 & 0 & 26.53310155 & 77 & 39 & 2.457627119 \\
\hline The effect of modification & $\begin{array}{c}\text { No parameter } \\
\text { changes }\end{array}$ & $\begin{array}{c}\text { Improvement of } \\
\text { the parameter }\end{array}$ & $\begin{array}{c}\text { No parameter } \\
\text { changes }\end{array}$ & $\begin{array}{c}\text { No parameter } \\
\text { changes }\end{array}$ & $\begin{array}{c}\text { Deterioration of } \\
\text { the parameter }\end{array}$ \\
\hline
\end{tabular}

Source: the author's own elaboration based on tables 3, 5 and 7.

As shown in Table 8, the modifications had a positive impact on the change in the average path length, so the implementation of the proposed modification to a small extent contributes to the improvement of its parameters, which in the future could translate into an increase in the number of travelers and consequently have a positive impact on the financial results of the company. The introduced modifications also increase the transport accessibility of the carrier's transport network, which is important in terms of providing a better level of customer service, and this is one of the most important elements of managing a service enterprise [Witkowski 1995].

\section{Summary}

The analysed network of connections of Koleje Małopolskie is intended to enable the implementation of efficient and useful connections in the area of the Kraków agglomeration and the entire voivodship. The analyses carried out using the graph theory showed that the 
network enables communication on the main relations in the voivodship (especially within the Kraków agglomeration). The proposed modifications do not significantly affect the improvement of network parameters, but they also include proposals for connections via highly urbanized and populated areas of Kraków (including Nowa Huta).

The introduction of these connections may contribute to improving the carrier's offer, which may translate into changing the transport habits of at least some residents (change of means of transport from an individual vehicle to public transport) and what will be very important is the availability of transport in the analysed area. It may contribute to very important and necessary changes related to, among others, reduced congestion in the city and the emission of harmful substances. An important element of such a process will also increase the importance of the carrier on the local market of public transport services and it will probably cause a further increase in the number of travelers, which should also translate into the company's financial results and strengthening its market position.

The above proposal to add new nodes to the existing connection network may contribute to the competitiveness of the carrier, as well as the entire network of its connections will then improve (which has been demonstrated as part of the analyses and simulations and the results obtained, among others, a slight improvement of the average path length).

According to the author, the Koleje Małopolskie railway carrier should consider the implementation of the analyses into its activities related to investments or development of the network, e.g. using the graphs used in the article. This will allow obtaining additional information about the parameters of the proposed network (or its modification), which information may contribute to reducing the risk of introducing negative changes in the network of connections.

Undoubtedly, the conducted and proposed analysis of connection networks using the graph theory does not exhaust the issue and the factors necessary to be taken into account while managing the company when making key decisions. The afore-mentioned series of factors (including technical parameters of individual connection sections between nodes) are not taken into account, however, they were not the purpose of the article. As it was shown, the aim of the article was to analyse the carrier's connection network and propose this analysis as another element that is part of the process of making key decisions in the company.

\section{References}

1. Amaral LAN, Scala A, Barthelemy M, Stanley HE., 1997. Classes of small-world net- works. Proc Natl Acad Sci USA2000; (21):11149-52.

2. Arenas A., Danon L., Diaz-Guilera A., Gleiser P.M., Guimera R., 2003. Community analysis in social networks. Eur Phys JB, Vol. 38(2), pp. 373-80.

3. Bullmore E., Sporns O., 2009. Complex brain networks: graph theoretical analysis of structural and functional systems. Nat Rev Neuro Sci, vol. 10(3), pp. 186-98. 
4. Dunn S., Wilkinson S., 2017. Hazard tolerance of spatially distributed complex networks. Reliability Engineering and System Safety 157 1-12, Elsevier.

5. Li H, Guo XM, Xu Z, Hu XB., 2014. A study on the spatial vulnerability of the civil aviation network system in China, Qingdao: Proceedings of the IEEE 17th international conference on intelligent transportation systems, China.

6. Newman M.E.J., 2010. Networks: An Introduction. New York: Oxford University Press Inc.

7. Newman MEJ, Watts DJ, Strogatz SH., 2002. Random graph models of social net- works. Proc Natl Acad Sci USA, 99, 2566-72.

8. Ouyang M, Pan Z, Hong L, He Y., 2015. Vulnerability analysis of complementary transportation systems with applications to railway and airline systems in China. Reliab Eng Syst Saf, 142:248.

9. Rual J.-F., Venkatesan K., Hao T., Hirozane-Kishikawa T., Dricot A., Li N., Berriz G.F., Gibbons F.D., Dreze M., Ayivi-Guedehoussou N., Klitgord N., Simon C., Boxem M., Milstein S., Rosenberg J., Goldberg D.S., Zhang L.V., Wong S.L., Franklin G., Li S., Albala J.S., Lim J., Fraughton C., Llamosas E., Cevik S., Bex C., Lamesch P., Sikorski R.S., Vandenhaute J., Zoghbi H.Y., Smolyar A., Bosak S., Sequerra R., Doucette-Stamm L., Cusick M.E., Hill D.E., Roth F.P., Vidal M., 2005. Towards a proteome-scale map of the human protein - protein interaction network. Nature, Vol. 437(7062).

10. Sporns O., 2002. Network analysis, complexity, and brain function. Complexity, Vol. 8(1), pp. 56-60.

11. Stam C.J., Reijneveld J.C., 2007. Graph theoretical analysis of complex networks in the brain. Nonlinear Biomed Phys, Vol. 1(3), pp. 1-19.

12. Tarapata Z., 2015. Modelling and analysis of transportation networks using complex networks: Poland case study. The Archives of Transport, Vol. 36, Issue 4.

13. Valverde S., Solé R.V., 2003. Hierarchical small worlds in software architecture. Arxiv Prepr Cond-Mat/0307278.

14. Wilkinson S, Dunn S, Ma S., 2012. The vulnerability of the European air traffic network to spatial hazards. Nat Hazards, 60(3), 1027-36.

15. Witkowski J., 1995. Strategia logistyczna przedsiębiorstw przemysłowych. Wrocław: Wydawnictwo Akademii Ekonomicznej we Wrocławiu.

16. malopolskiekoleje.pl/ [accessed: 28.03.2018].

17. Statistical data received electronically from the Office of Rail Transport on January 16, 2018. 
